\title{
ON THE RELATIVE REFLEXIVITY OF FINITELY GENERATED MODULES OF OPERATORS
}

\author{
BOJAN MAGAJNA
}

\begin{abstract}
Let $\mathscr{R}$ be a von Neumann algebra on a Hilbert space $\mathscr{H}$ with commutant $\mathscr{R}^{\prime}$ and centre $\mathscr{C}$. For each subspace $\mathscr{S}$ of $\mathscr{R}$ let $\operatorname{ref}_{\mathscr{R}}(\mathscr{S})$ be the space of all $B \in \mathscr{R}$ such that $X B Y=0$ for all $X, Y \in \mathscr{R}$ satisfying $X \mathscr{S} Y=0$. If $\operatorname{ref}_{\mathscr{R}}(\mathscr{S})=\mathscr{S}$, the space $\mathscr{S}$ is called $\mathscr{R}$-reflexive. (If $\mathscr{R}=$ $\mathscr{B}(\mathscr{H})$ and $\mathscr{S}$ is an algebra containing the identity operator, $\mathscr{R}$-reflexivity reduces to the usual reflexivity in operator theory.) The main result of the paper is the following: if $\mathscr{S}$ is one-dimensional, or if $\mathscr{S}$ is arbitrary finitedimensional but $\mathscr{R}$ has no central portions of type $\mathrm{I}_{n}$ for $n>1$, then the space $\overline{\mathscr{C S}}$ is $\mathscr{R}$-reflexive and the space $\overline{\mathscr{R}^{\prime} \mathscr{S}}$ is $\mathscr{B}(\mathscr{H})$-reflexive, where the bar denotes the closure in the ultraweak operator topology. If $\mathscr{R}$ is a factor, then $\mathscr{R}^{\prime} \mathscr{S}$ is closed in the weak operator topology for each finite-dimensional subspace $\mathscr{S}$ of $\mathscr{R}$.
\end{abstract}

\section{INTRODUCTION, NOTATION AND STATEMENT OF THE MAIN RESULT}

Throughout this paper $\mathscr{H}$ denotes a Hilbert space, $\mathscr{B}(\mathscr{H})$ the algebra of all bounded operators on $\mathscr{H}, \mathscr{R}$ a von Neumann subalgebra of $\mathscr{B}(\mathscr{H}), \mathscr{R}^{\prime}$ the commutant of $\mathscr{R}$, and $\mathscr{C}$ the centre of $\mathscr{R}$. The set of all projections in $\mathscr{R}$ (that is, selfadjoint idempotents) is denoted by $\operatorname{Proj}(\mathscr{R})$, and for each projection $P$ the projection $I-P$ is denoted by $P^{\perp}$, where $I$ is the identity operator on $\mathscr{H}$. The closure of any subset $\mathscr{T}$ of $\mathscr{B}(\mathscr{H})$ in the ultraweak $(=\sigma$ weak) operator topology is denoted by $\overline{\mathscr{T}}$, and for any subset $\mathscr{K}$ of $\mathscr{H}$ the symbol $[\mathscr{T} \mathscr{H}]$ stands for the closure of the linear span of the set $\{T x: T \in \mathscr{T}$, $x \in \mathscr{K}\}$. For each cardinal $n, \mathscr{H}^{n}$ is the direct sum of $n$ copies of $\mathscr{H}$, $M_{n}(\mathscr{R})$ is the von Neumann algebra of all those bounded operators on $\mathscr{H}^{n}$ that can be represented by matrices with entries in $\mathscr{R}$, and $\mathscr{R}^{(n)}$ is the subalgebra consisting of all those diagonal matrices that have the same element from $\mathscr{R}$ along the diagonal. For each $T \in \mathscr{R}$ the diagonal matrix with $T$ along the diagonal is denoted by $T^{(n)}$.

Received by the editors August 11, 1989.

1980 Mathematics Subject Classification (1985 Revision). Primary 47D15, 47D25; Secondary 46L99.

Key words and phrases. Subspaces of operators, reflexivity, elementary operators, von Neumann algebras.

Supported in part by the Republic of Slovenia Science Foundation. 
A subalgebra $\mathscr{S}$ of $\mathscr{B}(\mathscr{H})$ is called reflexive if it contains all operators in $\mathscr{B}(\mathscr{H})$ that leave invariant all closed subspaces of $\mathscr{H}$ which are invariant for $\mathscr{S}$. Let us identify each closed subspace $\mathscr{K}$ of $\mathscr{H}$ with the orthogonal projection onto $\mathscr{K}$, denote $\mathscr{R}=\mathscr{B}(\mathscr{H})$ and put

$$
\operatorname{ref}_{\mathscr{R}}(\mathscr{S})=\left\{B \in \mathscr{R}:(\forall P \in \operatorname{Proj}(\mathscr{R})) P^{\perp} \mathscr{S} P=0 \Rightarrow P^{\perp} B P=0\right\} .
$$

Then $\mathscr{S}$ is reflexive if and only if $\operatorname{ref}_{\mathscr{R}}(\mathscr{S})=\mathscr{S}$. If $\mathscr{S}$ is generated by operators $A$ and $I$, then the reflexivity of $\mathscr{S}$ implies that $A$ has nontrivial invariant subspaces. This has been perhaps the first main reason to study reflexive algebras; however, reflexivity is important also for other reasons, as can be seen, for example, in monographs [3] and [8]. Loginov and Sulman [20, 26] extended the notion of reflexivity from algebras to subspaces of operators. For each subspace $\mathscr{S}$ of the algebra $\mathscr{R}=\mathscr{B}(\mathscr{H})$ let

$$
\operatorname{ref}_{\mathscr{R}}(\mathscr{S})=\{B \in \mathscr{R}:(\forall P, Q \in \operatorname{Proj}(\mathscr{R})) P \mathscr{S} Q=0 \Rightarrow P B Q=0\} .
$$

Then $\mathscr{S}$ is reflexive in the sense of Loginov and Sulman iff $\operatorname{ref}_{\mathscr{R}}(\mathscr{S})=\mathscr{S}$. It is easy to verify that a subalgebra containing the identity $I$ is reflexive as a subalgebra if and only if it is reflexive as a subspace. Reflexive subspaces have been used by Kraus and Larson $[15,16]$ to construct reflexive algebras and to study questions related to the Arveson distance formula. A nice exposition of reflexivity of spaces and many examples of reflexive and nonreflexive subspaces can be found in [5].

Obviously the same definition of reflexivity makes sense for subspaces of algebras more general than $\mathscr{B}(\mathscr{H})$, but since it is possible for an algebra to have only trivial projections, it is more natural to replace the projections $P$ and $Q$ in the definition by arbitrary elements. So we arrive at the following definition, which has been proposed in [17].

Definition. For each (complex) algebra $\mathscr{A}$ and each subset $\mathscr{S}$ of $\mathscr{A}$ let

$$
\operatorname{ref}_{\mathscr{A}}(\mathscr{S})=\{B \in \mathscr{A}:(\forall X, Y \in \mathscr{A}) X \mathscr{S} Y=0 \Rightarrow X B Y=0\}
$$

A linear subspace $\mathscr{S}$ of $\mathscr{A}$ is called $\mathscr{A}$-reflexive (or reflexive relative to $\mathscr{A}$ ) iff $\operatorname{ref}_{\mathscr{A}}(\mathscr{S})=\mathscr{S}$.

For each $T \in \mathscr{B}(\mathscr{H})$ we denote by $R(T)$ the range projection of $T$ (that is, the projection onto $[T \mathscr{H}]$ ), and by $N(T)$ the null projection of $T$ (the projection onto $\operatorname{Ker} T$ ). Since a von Neumann algebra $\mathscr{R}$ contains $R(T)$ and $N(T)$ for each $T \in \mathscr{R}$, and since for any $X, Y, T \in \mathscr{R}$ the identity $X T Y=0$ is equivalent to $N(X)^{\perp} T R(Y)=0$, we see that for subspaces of von Neumann algebras, $\operatorname{ref}_{\mathscr{R}}(\mathscr{S})$ can be defined by (1.2) instead of (1.3). In this form the relative reflexivity has already appeared before, for instance in [19].

More generally, for a subspace $\mathscr{S}$ of $\mathscr{B}(\mathscr{H})$ and a von Neumann algebra $\mathscr{R}$ on $\mathscr{H}$ which does not necessarily contain $\mathscr{S}$, we can consider the set

$$
\mathscr{S}_{\mathscr{R}}=\{B \in \mathscr{B}(\mathscr{H}):(\forall P, Q \in \operatorname{Proj}(\mathscr{R})) P \mathscr{S} Q=0 \Rightarrow P B Q=0\} .
$$


(If $\mathscr{S}$ is a subspace of $\mathscr{R}$, then $\operatorname{ref}_{\mathscr{R}}(\mathscr{S})=\mathscr{S}_{\mathscr{R}} \cap \mathscr{R}$.) Suppose that $\mathscr{S}$ is an $\mathscr{R}^{\prime}$-bimodule, that is, $\mathscr{R}^{\prime} \mathscr{S}=\mathscr{S}$ and $\mathscr{S} \mathscr{R}^{\prime}=\mathscr{S}$. Then for each $x \in \mathscr{H}$ the two projections $P_{x}$ with range $[\mathscr{S} x]^{\perp}$ and $Q_{x}$ with range $\left[\mathscr{R}^{\prime} x\right]$ are in $\mathscr{R}$ (since their ranges are invariant under $\mathscr{R}^{\prime}$ ), and $P_{x} \mathscr{S} Q_{x}=0$. Thus, for eachi $B \in \mathscr{S}_{\mathscr{R}}$ we have $P_{x} B Q_{x}=0$, hence $B x \in[\mathscr{S} x]$. Conversely, if $B \in \mathscr{B}(\mathscr{H})$ is such that $B x \in[\mathscr{S} x]$ for all $x \in \mathscr{H}$, then $B \in \mathscr{S}_{\mathscr{R}}$. Indeed, suppose that $P, Q \in \operatorname{Proj}(\mathscr{R})$ satisfy $P \mathscr{S} Q=0$. Then for each $x \in Q \mathscr{H}$ the relation $P[\mathscr{S} x] \subseteq P[\mathscr{S} Q \mathscr{H}]=0$ implies $P B x=0$ (since $B x \in[\mathscr{S} x])$, hence we have $P B Q=0$ and therefore $B \in \mathscr{S}_{\mathscr{R}}$. We have just seen that $\mathscr{S}_{\mathscr{R}}=\{B \in \mathscr{B}(\mathscr{H}): B x \in[\mathscr{S} x] \forall x \in \mathscr{H}\}$. The last set is independent of von Neumann algebra $\mathscr{R}^{\prime}$ over which $\mathscr{S}$ is a bimodule, hence $\mathscr{S}_{\mathscr{R}}=\mathscr{S}_{\mathscr{B}(\mathscr{H})}=\operatorname{ref}_{\mathscr{B}(\mathscr{H})}(\mathscr{S})$. This simple observation will be used later several times, so we formulate it as a lemma.

Lemma 1.1. If $\mathscr{S}$ is an $\mathscr{R}^{\prime}$-bimodule in $\mathscr{B}(\mathscr{H})$, then

$$
\operatorname{ref}_{\mathscr{B}(\mathscr{H})}(\mathscr{S})=\{B \in \mathscr{B}(\mathscr{H}):(\forall P, Q \in \operatorname{Proj}(\mathscr{R})) P \mathscr{S} Q=0 \Rightarrow P B Q=0\} .
$$

An elementary operator of length less than or equal to $n$ on $\mathscr{B}(\mathscr{H})$ is a map $\varphi: \mathscr{B}(\mathscr{H}) \rightarrow \mathscr{B}(\mathscr{H})$ of the form

$$
\varphi(T)=\sum_{i=1}^{n} A_{i} T B_{i} \quad(T \in \mathscr{B}(\mathscr{H})),
$$

where $A_{i}$ and $B_{i}$ are fixed elements of $\mathscr{B}(\mathscr{H})$. If $A_{i}$ and $B_{i}$ are all in $\mathscr{R}$, then clearly $\varphi$ is an $\mathscr{R}^{\prime}$-bimodule homomorphism of $\mathscr{B}(\mathscr{H})$. Lemma 1.1 has as a consequence the following Hahn-Banach type result.

Corollary 1.2. Let $\mathscr{S}$ be a subspace of $\mathscr{B}(\mathscr{H})$, closed in the weak operator topology, which is at the same time a bimodule over a von Neumann subalgebra $\mathscr{R}$ of $\mathscr{B}(\mathscr{H})$. Then for each $B \in \mathscr{B}(\mathscr{H}) \backslash \mathscr{S}$ there exists an $\mathscr{R}$-bimodule homomorphism $\varphi: \mathscr{B}(\mathscr{H}) \rightarrow \mathscr{B}(\mathscr{H})$ of the form (1.4) such that $\varphi(\mathscr{S})=0$ and $\varphi(B) \neq 0$.

Proof. Let $\omega$ be a weak-operator continuous linear functional on $\mathscr{B}(\mathscr{H})$ such that $\omega(\mathscr{S})=0$ and $\omega(B) \neq 0$. Then there exist $x_{i}, y_{i} \in \mathscr{H}, i=1, \ldots, n$, such that

$$
\omega(T)=\sum_{i=1}^{n}\left\langle T x_{i}, y_{i}\right\rangle
$$

for each $T \in \mathscr{B}(\mathscr{H})$. With $x=\left(x_{1}, \ldots, x_{n}\right)$ and $y=\left(y_{1}, \ldots, y_{n}\right) \in \mathscr{H}^{n}$ we have $\omega(T)=\left\langle T^{(n)} x, y\right\rangle$. From $\omega(\mathscr{S})=0$ and $\omega(B) \neq 0$ we have that $y$ is orthogonal to $\left[\mathscr{S}^{(n)} x\right]$ and that $\left\langle B^{(n)} x, y\right\rangle \neq 0$, hence $B^{(n)} x \notin\left[\mathscr{S}^{(n)} x\right]$. This implies that $B^{(n)} \notin \operatorname{ref}_{\mathscr{B}\left(\mathscr{K}^{n}\right)}\left(\mathscr{S}^{(n)}\right)$. Since $\mathscr{S}^{(n)}$ is an $\mathscr{R}^{(n)}$-bimodule, by Lemma 1.1 there exist two projections $P^{\prime}$ and $Q^{\prime}$ in $\left(\mathscr{R}^{(n)}\right)^{\prime}=M_{n}\left(\mathscr{R}^{\prime}\right)$ such that $P^{\prime} \mathscr{S}^{(n)} Q^{\prime}=0$ and $P^{\prime} B^{(n)} Q^{\prime} \neq 0$. If $A_{i}^{\prime}$ are elements of any row of $P^{\prime}$ and $B_{i}^{\prime}$ are elements of any column of $Q^{\prime}$, and if $\varphi$ is defined by (1.4) with 
$A_{i}^{\prime}$ and $B_{i}^{\prime}$ instead of $A_{i}$ and $B_{i}$, then $\varphi(\mathscr{S})=0$. By choosing the row and the column appropriately, we have also $\varphi(B) \neq 0$ (since $P^{\prime} B^{(n)} Q^{\prime} \neq 0$ ).

Clearly the bimodule $\mathscr{S}$ in Corollary 1.2 is reflexive precisely when for each $B \in \mathscr{B}(\mathscr{H})$ the homomorphism $\varphi$ can be chosen so that it is represented by an elementary operator of length one.

If $\mathscr{S}$ is a subalgebra of $\mathscr{R}$ containing $I$, then $\operatorname{ref}_{\mathscr{R}}(\mathscr{S})$ can be defined by (1.1), as in the case $\mathscr{R}=\mathscr{B}(\mathscr{H})$. (We omit the simple proof of this fact which will not be used in next sections.) If, in addition, $\mathscr{S}$ is selfadjoint, then for a projection $P \in \mathscr{R}$ the condition $P^{\perp} \mathscr{S} P=0$ is equivalent to the condition that $P$ commutes with $\mathscr{S}$, and the space $\operatorname{ref}_{\mathscr{R}}(\mathscr{S})$ coincides with the relative double commutant of $\mathscr{S}$ in $\mathscr{R}$, that is, $\operatorname{ref}_{\mathscr{R}}(\mathscr{S})=\left(\mathscr{S}^{\prime} \cap \mathscr{R}\right)^{\prime} \cap \mathscr{R}$. The question when the relative double commutant in $\mathscr{R}$ of each von Neumann subalgebra $\mathscr{S}$ of $\mathscr{R}$ coincides with $\mathscr{S}$ has already been considered by Murray and von Neumann [24]. Von Neumann algebras $\mathscr{R}$ satisfying the condition $\operatorname{ref}_{\mathscr{R}}(\mathscr{S})=\mathscr{S}$ for each von Neumann subalgebra $\mathscr{S} \subseteq \mathscr{R}$ are called normal. Now it is known that normal von Neumann algebras on separable spaces are precisely factors of type I [14, p. 1046]. There are examples of factors $\mathscr{R}$ containing subfactors $\mathscr{S}$ such that $\mathscr{S}^{\prime} \cap \mathscr{R}=\mathbb{C} I$ [14, p. 927]; in this case we have $\operatorname{ref}_{\mathscr{R}}(\mathscr{S})=(\mathbb{C} I)^{\prime} \cap \mathscr{R}=\mathscr{R}$. These are at the same time examples of subspaces of $\mathscr{R}$ which are reflexive in $\mathscr{B}(\mathscr{H})$, but not relatively reflexive in $\mathscr{R}$.

In this article we shall investigate the relative reflexivity of finitely generated central submodules over $\mathscr{R}$ and at the same time the reflexivity of $\mathscr{R}^{\prime}$ submodules of $\mathscr{B}(\mathscr{H})$ generated by finite subsets of $\mathscr{R}$. The main result is the following.

Theorem 1.3. Let $\mathscr{R}$ be a countably generated von Neumann algebra on a Hilbert space $\mathscr{H}$ with centre $\mathscr{C}$ and let $\mathscr{S}$ be a finite-dimensional subspace of $\mathscr{R}$. If $\mathscr{S}$ is one-dimensional, or if $\mathscr{R}$ has no central portions of type $\mathrm{I}_{n}$ for $n>1$, then:

(i) $\operatorname{ref}_{\mathscr{B}(\mathscr{H})}\left(\mathscr{R}^{\prime} \mathscr{S}\right)=\overline{\mathscr{R}^{\prime} \mathscr{S}}$ and

(ii) $\operatorname{ref}_{\mathscr{R}}(\mathscr{S})=\overline{\mathscr{C} \mathscr{S}}$.

It is easy to show that if $\mathscr{R}$ contains a central portion of type $\mathrm{I}_{n}$ for some cardinal $n>1$, then there is a finite-dimensional subspace $\mathscr{S}$ in $\mathscr{R}$ such that $\mathscr{R}^{\prime} \mathscr{S}$ is not $\mathscr{B}(\mathscr{H})$-reflexive and $\overline{\mathscr{C} S}$ is not $\mathscr{R}$-reflexive (see the remark after the proof of Theorem 1.3 in $\S 4$ ). The reason for the restriction to countably generated algebras in this theorem is the use of the direct integral decomposition in the proof (such a decomposition requires a separable Hilbert space). If $\mathscr{R}$ is a factor, then there is no countability restriction in the proof, and in addition we shall see that in this case $\mathscr{R}^{\prime} \mathscr{S}$ is closed in the weak operator topology.

If $\mathscr{R}=\mathscr{B}(\mathscr{H})$, we obtain from this theorem, as a special case, the known fact that each one-dimensional subspace of $\mathscr{B}(\mathscr{H})$ is reflexive [15, Lemma 10]. The theorem is motivated by the recent work of Larson [18], where it is proved 
in particular that each finite-dimensional subspace of $\mathscr{B}(\mathscr{H})$, which does not contain nonzero operators of finite rank, is necessarily reflexive. Partially it is also motivated by a result irom [21], which implies that each finite-dimensional subspace of the Calkin algebra is reflexive.

In $\S 2$ we shall first show that the question of reflexivity of $\mathscr{C}$-modules (or $\mathscr{R}^{\prime}$-modules) generated by a single operator is connected with a certain operator equation, which has been studied in the algebra $\mathscr{B}(\mathscr{H})$ by Fong and Sourour [10] and in prime $C^{*}$-algebras by Mathieu [23]. Then Theorem 1.3 will be proved in the special case when $\mathscr{S}$ is one-dimensional. In fact, a slightly more general result in the context of $C^{*}$-algebras will be proved, which has some interesting applications. In $\S 3$ the case of factors will be studied, and in $\S 4$ the proof of Theorem 1.3 will be completed by reduction to the case of factors. $\S 4$ also contains a consideration of $n$-reflexivity.

From now on we shall denote $\operatorname{ref}_{\mathscr{B}(\mathscr{H})}(\mathscr{S})$ simply by $\operatorname{ref}(\mathscr{S})$ and the word "reflexive" will always mean " $\mathscr{B}(\mathscr{H})$-reflexive".

\section{SINGLY GENERATED MODULES}

The following lemma might be known to specialists in operator algebras and is similar to a result concerning the algebraic tensor product of $\mathscr{R}$ and $\mathscr{R}^{\prime}$ (see [14, 24 or 27]), but we have not found any reference for it, so a short proof is included.

Lemma 2.1. Let $\mathbf{A}=\left(A_{1}, \ldots, A_{n}\right)$ and $\mathbf{B}=\left(B_{1}, \ldots, B_{n}\right)$ be two $n$-tuples of elements of $\mathscr{B}(\mathscr{H})$ and let $\mathscr{R}$ be a von Neumann algebra on $\mathscr{H}$. Then the identity

$$
\sum_{i=1}^{n} A_{i} T B_{i}=0
$$

holds for all $T \in \mathscr{R}$ if and only if there exists a projection $P^{\prime}=\left[C_{i j}^{\prime}\right] \in M_{n}\left(\mathscr{R}^{\prime}\right)$ such that

$$
\sum_{i=1}^{n} A_{i} C_{i j}^{\prime}=0 \quad \text { and } \quad B_{i}=\sum_{j=1}^{n} C_{i j}^{\prime} B_{j}
$$

for all $i, j \in\{1, \ldots, n\}$. Moreover, if $\mathbf{B} \in \mathscr{R}^{n}$, then $P^{\prime}$ can be chosen to be in $M_{n}(\mathscr{C})$.

Proof. Let $\tilde{A} \in M_{n}(\mathscr{B}(\mathscr{H}))=\mathscr{B}\left(\mathscr{H}^{n}\right)$ be the matrix with the first row equal to $\mathbf{A}$ and the remaining rows identically zero, let $\widetilde{B} \in M_{n}(\mathscr{B}(\mathscr{H}))$ be the matrix with the first column equal to the transpose of $\mathbf{B}$ and the remaining columns equal to zero, and recall that $T^{(n)}$ is the diagonal matrix with $T$ along the diagonal. Then the identity $(2.1)$ can be written as $\widetilde{A} T^{(n)} \widetilde{B}=0$. Let $P^{\prime}=\left[C_{i j}^{\prime}\right] \in \mathscr{B}\left(\mathscr{H}^{n}\right)$ be the orthogonal projection onto $\left[\mathscr{R}^{(n)} \widetilde{B} \mathscr{C}^{n}\right]$. Since $\widetilde{A} T^{(n)} \widetilde{B}=0$ for all $T \in \mathscr{R}$, we have $\widetilde{A} P^{\prime}=0$. From the definition of $P^{\prime}$ we also have $P^{\prime} \widetilde{B}=\widetilde{B}$. The last two identities (when written componentwise) 
are just the identities (2.2). Since the range of $P^{\prime}$ is invariant under $\mathscr{R}^{(n)}, P^{\prime}$ must be in $\left(\mathscr{R}^{(n)}\right)^{\prime}=M_{n}\left(\mathscr{R}^{\prime}\right)$.

If $\mathbf{B} \in \mathscr{R}^{n}$, then $\widetilde{B} \in M_{n}(\mathscr{R})$, hence $\widetilde{B}$ commutes with $\mathscr{R}^{(n)}$, the range of $P^{\prime}$ is invariant under $\mathscr{R}^{\prime(n)}$, and therefore $P^{\prime} \in\left(\mathscr{R}^{(n)}\right)^{\prime}=M_{n}(\mathscr{R})$. Thus, $P^{\prime} \in M_{n}\left(\mathscr{R}^{\prime}\right) \cap M_{n}(\mathscr{R})=M_{n}(\mathscr{C})$.

The converse, that (2.2) implies (2.1), can be shown by a direct computation.

Corollary 2.2. If $\mathscr{R}$ is a factor, $\mathbf{A}, \mathbf{B} \in \mathscr{R}^{n}$, and $A_{1}, \ldots, A_{n}$ are linearly independent, then (2.1) holds for all $T \in \mathscr{R}$ if and only if $B_{i}=0$ for all $i=1, \ldots, n$.

Proof. Since $\mathscr{R}$ is a factor, the central elements $C_{i j}^{\prime}$ in (2.2) are just the complex multiples of the identity operator $I$. Since the $A_{i}$ 's are linearly independent, the first family of equations (2.2) implies $C_{i j}^{\prime}=0$ for all $i, j$ and the second family then implies $B_{i}=0$ for all $i$.

Corollary 2.2 has been proved in the case $\mathscr{R}=\mathscr{B}(\mathscr{H})$ by Fong and Sourour [10] and recently it has been generalized to prime $C^{*}$-algebras by Mathieu [23]. The identity (2.1) has been originally considered by algebraists in prime rings [13, p. 22], where the answer involves the notion of the extended centroid, which is not always easy to compute for concrete rings.

Here we shall need an improvement of a special case of Lemma 2.1.

Lemma 2.3. Let $\mathscr{R}$ be a von Neumann algebra on $\mathscr{H}$ and $A, B \in \mathscr{B}(\mathscr{H})$. If $A T B=B T A$ for all $T \in \mathscr{R}$, then there exists a projection $Q^{\prime} \in \mathscr{R}^{\prime}$ such that $Q^{\prime} B \in \overline{\mathscr{R}^{\prime} A}$ and $A Q^{\perp}=0$. If, in addition, $A, B \in \mathscr{R}$, then $Q^{\prime}$ can be chosen in $\mathscr{C}$ and $Q^{\prime} B \in \overline{\mathscr{C} A}$.

Proof. Since $A T B+B T(-A)=0$ for all $T \in \mathscr{R}$, Lemma 2.1 gives us a projection

$$
P^{\prime}=\left[\begin{array}{ll}
C_{1}^{\prime} & C_{2}^{\prime} \\
C_{2}^{\prime *} & C_{3}^{\prime}
\end{array}\right]
$$

in $M_{2}\left(\mathscr{R}^{\prime}\right)$ such that

$$
A C_{1}^{\prime}+B C_{2}^{\prime *}=0, \quad A C_{2}^{\prime}+B C_{3}^{\prime}=0
$$

and

$$
B=C_{1}^{\prime} B-C_{2}^{\prime} A, \quad-A=C_{2}^{\prime *} B-C_{3}^{\prime} A .
$$

From (2.4) we obtain

$$
\left(I-C_{1}^{\prime}+C_{2}^{\prime} C_{2}^{\prime *}\right) B=C_{2}^{\prime}\left(C_{3}^{\prime}-2 I\right) A .
$$

Let $Q^{\prime}$ be the range projection of $I-C_{1}^{\prime}+C_{2}^{\prime} C_{2}^{\prime *}$. By the functional calculus for selfadjoint operators there exists a sequence $\left(D_{n}^{\prime}\right)$ in $\mathscr{R}^{\prime}$ such that the sequence $D_{n}^{\prime}\left(I-C_{1}^{\prime}+C_{2}^{\prime} C_{2}^{\prime *}\right)$ converges to $Q^{\prime}$ ultrastrongly, hence $(2.5)$ implies 
that $Q^{\prime} B \in \overline{\mathscr{R}^{\prime} A}$. If in addition $A$ and $B$ are in $\mathscr{R}$, then $P^{\prime} \in M_{2}(\mathscr{C})$ by Lemma 2.1, hence $Q^{\prime} \in \mathscr{C}$ and (2.5) implies $Q^{\prime} B \in \overline{\mathscr{C} A}$.

We shall now verify that $A Q^{\perp}=0$, or equivalently, that $A\left(\operatorname{Ker} Q^{\prime}\right)=0$. Let $x \in \operatorname{Ker} Q^{\prime}$. Since $\operatorname{Ker} Q^{\prime}=\operatorname{Ker}\left(I-C_{1}^{\prime}+C_{2}^{\prime} C_{2}^{\prime *}\right)$ and since $I-C_{1}^{\prime}$ and $C_{2}^{\prime} C_{2}^{\prime *}$ are positive operators (for $P^{\prime}$ is a projection), it follows that $\left(I-C_{1}^{\prime}\right) x=0$ and $C_{2}^{\prime} C_{2}^{\prime *} x=0$. Thus we have $x=C_{1}^{\prime} x, C_{2}^{\prime *} x=0$, and from the first equation (2.3) we obtain $A x=0$.

The identity $A T B=B T A$ has been considered in the purely algebraic context of prime rings by Martindale [22]. In the special case when $\mathscr{R}$ is a factor Lemma 2.3 can be deduced also by combining [22, Theorem 1] with the result of Mathieu [23, Proposition 2.5] which states that the extended centroid of a prime $C^{*}$-algebra is isomorphic to the complex numbers.

Proposition 2.4. If $\mathscr{J}$ is a two-sided closed ideal of a $C^{*}$-algebra $\mathscr{A}$ and $\mathscr{S}$ is a linear subspace of $\mathcal{F}$, then

$$
\operatorname{ref}_{\mathscr{J}}(\mathscr{S})=\mathscr{J} \cap \operatorname{ref}_{\mathscr{A}}(\mathscr{S}) .
$$

Proof. Only the nontrivial inclusion $\operatorname{ref}_{\mathscr{J}}(\mathscr{S}) \subseteq \mathscr{J} \cap \operatorname{ref}_{\mathscr{A}}(\mathscr{S})$ will be proved here. Let $B \in \operatorname{ref}_{\mathscr{f}}(\mathscr{S})$. We must prove that $X B Y=0$ for all $X, Y \in$ $\mathscr{A}$ satisfying $X \mathscr{S} Y=0$. We may assume that $\mathscr{A}$ is contained in $\mathscr{B}(\mathscr{H})$ for some Hilbert space $\mathscr{H}$. Let $\left\{E_{n}\right\}$ be an approximate unit in $\mathscr{J}$ (such that $0 \leq E_{n} \leq I$ for all $n$, see [27, p. 27]). Then the net $\left\{E_{n}\right\}$ converges strongly to the projection $E$ in the centre of $\overline{\mathscr{A}}$ such that $\overline{\mathscr{J}}=E \overline{\mathscr{A}}$. From $\left(E_{n} X\right) \mathscr{S}\left(Y E_{n}\right)=0$ we have $E_{n} X B Y E_{n}=0$ (since $B \in \operatorname{ref}_{\mathscr{J}}(\mathscr{S})$ ), hence by taking the limit we obtain $E X B Y E=0$. Since $E$ is in the centre of $\overline{\mathscr{A}}$ and $B \in \mathscr{J}$, it follows that $X B Y=0$.

Let $\mathscr{A}$ be a $C^{*}$-subalgebra of $\mathscr{B}(\mathscr{H})$. A projection $P$ in $\overline{\mathscr{A}}$ is called open relative to $\mathscr{A}$ iff $P$ is contained in the weak operator closure of the algebra $P \mathscr{A} P \cap \mathscr{A}$; a projection $P \in \overline{\mathscr{A}}$ is closed relative to $\mathscr{A}$ if $P^{\perp}$ is open relative to $\mathscr{A}$. (In the case when $\mathscr{A}$ is the universal representation of some $C^{*}$. algebra several equivalent characterizations of open projections can be found in $[25$, p. 77 or 27, p. 168].) The central carrier of an element $A$ in a von Neumann algebra $\mathscr{R}$ is denoted by $C_{A}$. (By definition, $C_{A}$ is the smallest central projection in $\mathscr{R}$ satisfying $C_{A} A=A[14$, p. 333].)

Theorem 2.5. Let $\mathscr{A}$ be a $C^{*}$-subalgebra of $\mathscr{B}(\mathscr{H}), \mathscr{R}$ the ultraweak closure of $\mathscr{A}$, and $\mathscr{C}$ the centre of $\mathscr{R}$. Assume $I \in \mathscr{R}$. If $A \in \mathscr{A}$ is such that the projection $C_{A}$ is closed relative to $\mathscr{A}$, then $\operatorname{ref}_{\mathscr{A}}(A)=\overline{\mathscr{C} A} \cap \mathscr{A}$.

Proof. By Proposition 2.4 we may assume that $I \in \mathscr{A}$, since in general $\mathscr{A}$ is an ideal of $\mathscr{A}+\mathbb{C} I$. Clearly $\mathscr{A} \cap \overline{\mathscr{C} A} \subseteq \operatorname{ref}_{\mathscr{A}}(A)$, so only the reverse inclusion requires a proof. Let $B \in \operatorname{ref}_{\mathscr{A}}(A)$. Let $A=U|A|$ be the polar decomposition of $A$ in $\mathscr{R}$; note that $|A|^{t} U^{*} \in \mathscr{A}$ for each $t>0$ [1, Lemma 2.1]. Let $t>0$ be fixed, choose any positive element $H \in \mathscr{A}$, and let $E$ be any spectral projection of the positive operator $H|A|^{1+t} H$ corresponding to a closed or open subset 
of the spectrum. Using the spectral theorem it is easy to find two sequences $\left(S_{n}\right)$ and $\left(T_{n}\right)$ in the commutative $C^{*}$-algebra generated by $H|A|^{1+t} H$ and $I$, converging ultrastrongly to $E$ and $E^{\perp}$, respectively, such that $S_{n} T_{n}=0$ for all $n$. Then $\left(S_{n} H|A|^{t} U^{*}\right) A\left(H T_{n}\right)=S_{n} H|A|^{t+1} H T_{n}=0$, hence $S_{n} H|A|^{t} U^{*} B H T_{n}=$ 0 (since $B \in \operatorname{ref}_{\mathscr{A}}(A)$ ). By taking the limit in the last identity we obtain $E H|A|^{t} U^{*} B H E^{\perp}=0$. Comparing this equality with the one obtained by interchanging $E$ and $E^{\perp}$ we see that $E$ commutes with $H|A|^{t} U^{*} B H$. It follows (by the spectral theorem) that $H|A|^{1+t} H$ commutes with $H|A|^{t} U^{*} B H$ and, if $H$ is invertible, this implies $|A|^{1+t} H^{2}|A|^{t} U^{*} B=|A|^{t} U^{*} B H^{2}|A|^{1+t}$. Letting $t$ approach 0 in this equality, we obtain $|A| H^{2} U^{*} B=U^{*} B H^{2}|A|$. Since each element of $\mathscr{A}$ is a linear combination of positive invertible elements (which are necessarily of the form $H^{2}$ ), we have $|A| T U^{*} B=U^{*} B T|A|$ for all $T \in \mathscr{A}$ and, by continuity, for all $T \in \mathscr{R}$. By Lemma 2.3 there exists a projection $Q^{\prime} \in \mathscr{C}$ such that $Q^{\prime} U^{*} B \in \overline{\mathscr{C}|A|}$ and $|A| Q^{\perp}=0$. From $A Q^{\perp}=U|A| Q^{\perp}=0$ we have $C_{A} \leq Q^{\prime}$, hence $C_{A} U^{*} B \in \overline{\mathscr{C}|A|}$. Therefore we may assume $Q^{\prime}=C_{A}$ (otherwise just replace $Q^{\prime}$ by $C_{A}$ ). Put $P=Q^{\perp \perp}$. Since by hypothesis $C_{A}$ is closed, $P$ is open, hence (by the Kaplansky density theorem) there exists a bounded net of positive elements $G_{n}$ in $\mathscr{A} P \cap \mathscr{A}$ converging strongly to $P$. From $P A=Q^{\perp} A=0$ we have $G_{n} A=0$ (since $G_{n} \leq P$ ), hence $G_{n} B=0$ (since $B \in \operatorname{ref}_{\mathscr{A}}(A)$ ), and $P B=\lim G_{n} B=0$. This implies $P U^{*} B=0$ (since $P$ is central), hence we have now $U^{*} B=P^{\perp} U^{*} B=Q^{\prime} U^{*} B \in \overline{\mathscr{C}|A|}$, and this clearly implies that $B \in \overline{\mathscr{C} A}$.

Note that the condition of $C_{A}$ being closed relative to $\mathscr{A}$ is trivially satisfied for every invertible $A \in \mathscr{A}$. To see that this condition is not redundant in general, let $\mathscr{A}$ be the $C^{*}$-algebra of all continuous functions on the interval $\Lambda=[0,1]$, which act in the usual way (by multiplication) on the Hilbert space $L_{2}(\Lambda, \mu)$, where $\mu$ is the sum of the Lebesgue measure (restricted to $\Lambda$ ) and the point mass at 0 . The weak closure of $\mathscr{A}$ is identified with $L_{\infty}(\Lambda, \mu)=\mathscr{C}$. If $A$ is the (operator of multiplication by the) identity function (that is, $A(t)=t$ for each $t \in[0,1])$, then $\operatorname{ref}_{\mathscr{A}}(A)=\mathscr{A}$, but $\mathscr{A} \cap \overline{\mathscr{C} A}=\{B \in \mathscr{A}: B(0)=0\}$.

Corollary 2.6. Theorem 1.3 is true for one-dimensional spaces, that is, for each $A \in \mathscr{R}$ the following two identities hold:

(i) $\operatorname{ref}\left(\mathscr{R}^{\prime} A\right)=\overline{\mathscr{R}^{\prime} A}$;

(ii) $\operatorname{ref}_{\mathscr{R}}(A)=\overline{\mathscr{C} A}$.

Proof. The identity (ii) is just a special case of Theorem 2.5. The identity (i) is not a direct consequence of Theorem 2.5, but it can be proved in the same way. Indeed, the proof is simpler here, since $\mathscr{R}$ contains all spectral projections of its elements and since both factors $(U$ and $|A|)$ in the polar decomposition of $A$ are in $\mathscr{R}$, hence we can take in the previous proof $t=0, S_{n}=E$, and $T_{n}=E^{\perp}$ from the beginning. For a given $B \in \operatorname{ref}\left(\mathscr{R}^{\prime} A\right)$ we then obtain a 
projection $Q^{\prime} \in \mathscr{R}^{\prime}$ such that $Q^{\prime} U^{*} B \in \overline{\mathscr{R}^{\prime}|A|}$ and $|A| Q^{\perp}=0$. Since $U \in \mathscr{R}$, it follows $Q^{\prime} B \in \overline{\mathscr{R}^{\prime} A}$ and $Q^{\perp} \mathscr{R}^{\prime} A=Q^{\perp} A \mathscr{R}^{\prime}=0$; thus $Q^{\perp} B=0$ (since $B \in \operatorname{ref}\left(\mathscr{R}^{\prime}(A)\right)$ and $B=Q^{\prime} B \in \overline{\mathscr{R}^{\prime} A}$.

Corollary 2.7. If $\mathscr{A}$ is an irreducible $C^{*}$-algebra of operators on a Hilbert space $\mathscr{H}$, then each one-dimensional subspace of $\mathscr{A}$ is relatively reflexive in $\mathscr{A}$.

Proof. Since $\mathscr{A}$ is irreducible, $\overline{\mathscr{A}}=\mathscr{B}(\mathscr{H})$ and $C_{A}=I$ for each $A \neq 0$, so Theorem 2.5 applies.

Another immediate consequence of Theorem 2.5 is the known result of Kaplansky that each noncommutative $C^{*}$-algebra contains nonzero nilpotents [14, p. 292]. In fact a slightly stronger result is true.

Corollary 2.8. Let $\mathscr{A}$ be a $C^{*}$-algebra with centre $\mathscr{C}(\mathscr{A})$. For each $A \in$ $\mathscr{A} \backslash \mathscr{C}(\mathscr{A})$ there exist positive elements $X, Y$ in $\mathscr{A}$ such that $X A Y \neq 0$ and $(X A Y)^{2}=0$.

Proof. Assume first that $\mathscr{A}$ has a unit; then we may suppose that $\mathscr{A}$ is embedded in $\mathscr{B}(\mathscr{H})$ and $I \in \mathscr{A}$. From Theorem 2.5 we have $\operatorname{ref}_{\mathscr{A}}(I)=\mathscr{C}(\mathscr{A})$, hence $A \notin \operatorname{ref}_{\mathscr{A}}(I)$. Therefore there exist $X, Y \in \mathscr{A}$ such that $X Y=0$ and $X A Y \neq 0$. We may suppose that $X$ and $Y$ are positive (otherwise we replace $X$ and $Y$ by $|X|$ and $\left|Y^{*}\right|$, respectively, using the polar decompositions in $\mathscr{B}(\mathscr{L}))$. Then $X Y=0$ implies $Y X=0$, hence $(X A Y)^{2}=0$.

If $\mathscr{A}$ is without unit, then let $\mathscr{A}$ be embedded in $\mathscr{B}(\mathscr{H})$ so that $I \in \overline{\mathscr{A}}$ and denote $\mathscr{A}_{I}=\mathscr{A}+\mathbb{C} I$. By the previous paragraph there exist $X, Y \in \mathscr{A}_{I}$ such that $X Y=0$ and $X A Y \neq 0$. Let $\left\{E_{n}\right\}$ be an approximate unit in $\mathscr{A}$ with $0 \leq E_{n} \leq I$. Then $\left(E_{n} X\right)\left(Y E_{n}\right)=0$ and there exists $n$ such that $E_{n} X A Y E_{n} \neq 0$, since $\left\{E_{n}\right\}$ converges strongly to $I$. Since $\mathscr{A}$ is a closed ideal in $\mathscr{A}_{I}$, the elements $X_{0}=\left|E_{n} X\right|$ and $Y_{0}=\left|E_{n} Y^{*}\right|$ are in $\mathscr{A}$, and as before we have $X_{0} A Y_{0} \neq 0$ and $\left(X_{0} A Y_{0}\right)^{2}=0$.

It is clear from (1.2) that for each $A \in \mathscr{R}$ we have $\operatorname{ref}_{\mathscr{R}}(A)=\{B \in \mathscr{R}$ : $(\forall Q \in \operatorname{Proj}(\mathscr{R})) B Q \mathscr{H} \subseteq[A Q \mathscr{H}]\}$, and by Corollary 2.6 this is equal to $\overline{\mathscr{C} A}$. The natural question now is, for a given $A \in \mathscr{R}$, which operators $B$ satisfy the condition $B Q \mathscr{H} \subseteq A Q \mathscr{H}$ for all $Q \in \operatorname{Proj}(\mathscr{R})$ ?

Proposition 2.9. Let $A \in \mathscr{R}$ and $B \in \mathscr{B}(\mathscr{H})$. The inclusion $B Q \mathscr{H} \subseteq A Q \mathscr{H}$ holds for all projections $Q \in \mathscr{R}$ if and only if $B \in A \mathscr{R}^{\prime}$. If in addition $B \in \mathscr{R}$, then $B \in \mathscr{C} A$.

Proof. If $B=A T^{\prime}$ for some $T^{\prime} \in \mathscr{R}^{\prime}$, then $B Q \mathscr{H}=A T^{\prime} Q \mathscr{H}=A Q T^{\prime} \mathscr{H} \subseteq$ $A Q \mathscr{H}$ for each $Q \in \mathscr{R}$. To prove the converse, let $B$ be such that $B Q \mathscr{H} \subseteq$ $A Q \mathscr{H}$ for all projections $Q$ in $\mathscr{R}$ and suppose first that $A$ is positive. Corollary 2.6 implies that $B \in \mathscr{R}^{\prime} A$ (if in addition $B \in \mathscr{R}$, then the same corollary implies $B \in \overline{\mathscr{C} A})$. Since $B \mathscr{H} \subseteq A \mathscr{H}$, there exists $D \in \mathscr{B}(\mathscr{H})$ such that $B=A D$. In fact, $D$ is defined by $D=\left(A \mid(\operatorname{Ker} A)^{\perp}\right)^{-1} B$, and $D$ is bounded by the closed graph theorem (see [9, Theorem 2.1 or 12]). (If in addition $B \in \mathscr{R}$, then an application of the double commutant theorem shows 
that $D$ is contained in the von Neumann algebra generated by $A$ and $B$, hence $D \in \mathscr{R}$.) If $E$ is a spectral projection of $A$ such that $A$ is bounded below on $E \mathscr{H}$, then $A D=B \in \overline{A \mathscr{R}^{\prime}}$ implies $E D \in \overline{E \mathscr{R}^{\prime}}=E \mathscr{R}^{\prime}$. (In the case when $B \in \mathscr{R}, A D \in \overline{\mathscr{C} A}$ implies $E D \in E \mathscr{C}$.) Since $E \mathscr{R}^{\prime}$ is just the commutant of $E \mathscr{R} E$ (and $E \mathscr{C}$ is just the centre of $E \mathscr{R} E$ [14, p. 335]), we have $(E D)(E T E)=(E T E)(E D)$ for each $T \in \mathscr{R}$. If we now choose for $E$ a projection from a sequence of spectral projections of $A$ converging strongly to the range projection $F$ of $A$, we obtain in the limit that $F D \in(F \mathscr{R} F)^{\prime}=F \mathscr{R}^{\prime}$. (In the case $B \in \mathscr{R}, D$ is in the commutative von Neumann algebra generated by $A$ and $B$, hence $D$ commutes with $F$ and it follows $F D=F D F \in \operatorname{centre}(F \mathscr{R} F)=F \mathscr{C}$.) Thus there exists $D^{\prime} \in \mathscr{R}^{\prime} \quad\left(D^{\prime} \in \mathscr{C}\right.$ if $\left.B \in \mathscr{R}\right)$ such that $F D=F D^{\prime}$ and we have now $B=A D=(A F) D=A F D^{\prime}=A D^{\prime}$, as was to be proved.

In general, when $A$ is not positive, let $A=U|A|$ be the polar decomposition. Since for each projection $Q$ in $\mathscr{R}$ the relation $B Q \mathscr{H} \subseteq A Q \mathscr{H}$ implies $U^{*} B Q \mathscr{H} \subseteq|A| Q \mathscr{H}$, it follows from the previous paragraph that $U^{*} B \in|A| \mathscr{R}^{\prime}$ (or $U^{*} B \in|A| \mathscr{C}$ if $\left.B \in \mathscr{R}\right)$, hence $B \in A \mathscr{R}^{\prime} \quad(B \in A \mathscr{C}$ if $B \in \mathscr{R})$.

To end the discussion of reflexivity of singly generated modules we give an example showing that $\operatorname{ref}\left(\mathscr{R}^{\prime} A\right)$ can be different from $\overline{\mathscr{R}^{\prime} A}$ for a general element $A \in \mathscr{B}(\mathscr{H})$, so the hypothesis of Corollary 2.6(i) that $A$ should be in $\mathscr{R}$ is not redundant.

Example 2.10. Let $\mathscr{K}=\mathbb{C}^{2}$ and let $\mathscr{J}$ be the two-dimensional subspace of $\mathscr{B}(\mathscr{K})$ generated by matrices $I$ and $N$, where

$$
N=\left[\begin{array}{ll}
0 & 1 \\
0 & 0
\end{array}\right] \text {. }
$$

Let $\mathscr{H}=\mathscr{K}^{2}, \mathscr{R}=(\mathscr{B}(\mathscr{K}))^{(2)}$, and let $A \in \mathscr{B}(\mathscr{H})$ be given by the matrix

$$
A=\left[\begin{array}{ll}
I & 0 \\
N & 0
\end{array}\right] \text {. }
$$

An easy computation shows that $\mathscr{R}^{\prime} A$ consists of all operators in $\mathscr{B}(\mathscr{H})$ of the form

$$
\left[\begin{array}{ll}
T_{1} & 0 \\
T_{2} & 0
\end{array}\right]
$$

where $T_{1}, T_{2} \in \mathscr{J}$. By [15, Lemma 2] a general (weak* closed) subspace $\mathscr{S}$ of $\mathscr{B}(\mathscr{H})$ (where $\mathscr{H}$ is arbitrary) is reflexive if and only if the preannihilator $\mathscr{S}_{\perp}$ of $\mathscr{S}$ in the predual of $\mathscr{B}(\mathscr{H})$ is generated (as a Banach space) by its rank-1 operators. Now the preannihilator of $\mathscr{R}^{\prime} A$ consists of $2 \times 2$ matrices $\left[S_{i j}\right]$ with entries in $\mathscr{B}(\mathscr{K})$ such that $S_{11}$ and $S_{12}$ are in the preannihilator $\mathscr{J}_{\perp}$ of $\mathscr{J}$ in $\mathscr{B}(\mathscr{K})$. (Here the predual of $\mathscr{B}(\mathscr{K})$, which in general consists of trace class operators with the trace norm, has been identified as a set with $\mathscr{B}(\mathscr{K})$, since $\mathscr{K}$ is finite-dimensional.) A straightforward computation shows 
that $\mathscr{J}_{\perp}$ is not generated by its rank-1 elements and, consequently, $\left(\mathscr{R}^{\prime} A\right)_{\perp}$ is not generated by its rank-1 elements.

One might conjecture that the bimodule $\mathscr{R}^{\prime} A \mathscr{R}^{\prime}$ is reflexive in this case, but even this is false. Indeed, $\mathscr{R}^{\prime} A \mathscr{R}^{\prime}$ consists of all $2 \times 2$ matrices $\left[T_{i j}\right]$ with $T_{i j} \in \mathscr{J}$, hence $\left(\mathscr{R}^{\prime} A \mathscr{R}^{\prime}\right)_{\perp}$ consists of all $2 \times 2$ matrices $\left[S_{i j}\right]$ with $S_{i j} \in \mathscr{J}_{\perp}$ and is therefore not generated by its rank-1 elements.

We note that for certain von Neumann algebras $\mathscr{R}$ the spaces of the form $\overline{\mathscr{R}^{\prime} A}$ are reflexive for all $A \in \mathscr{B}(\mathscr{H})$. If, for example, $\mathscr{R}$ is an atomic maximal abelian selfadjoint subalgebra of $\mathscr{B}(\mathscr{H})$, then every weakly closed $\mathscr{R}$ submodule of $\mathscr{B}(\mathscr{H})$ is reflexive by [26].

\section{THE CASE OF FACTORS}

The main purpose of this section is to prove Theorem 1.3 in the case when $\mathscr{R}$ is a factor. For this we need some preparatory results.

Throughout the paper we shall denote by $\Omega$ the fundamental system of neighborhoods $\nu$ of 0 in the strong operator topology of $\mathscr{B}(\mathscr{H})$, defined by

$$
\nu=\left\{T \in \mathscr{B}(\mathscr{H}):\left\|T x_{i}\right\|<1, i=1, \ldots, n\right\},
$$

where $\left\{x_{1}, \ldots, x_{n}\right\}$ is any finite subset of $\mathscr{H}$.

The nontechnical part of the following lemma will be improved later (Corollary 3.6).

Lemma 3.1. Let $\mathscr{R} \subseteq \mathscr{B}(\mathscr{H})$ be a factor and let $\mathscr{S}$ be a finite-dimensional subspace of $\mathscr{R}$. Then:

(i) The subspace $\mathscr{R}^{\prime} \mathscr{S}$ is ultraweakly closed and for each ultraweakly dense subset $\mathscr{D}^{\prime}$ of $\mathscr{R}^{\prime}$ the set $\mathscr{D}^{\prime} \mathscr{S}$ is ultraweakly dense in $\mathscr{R}^{\prime} \mathscr{S}$.

(ii) If $B \in \mathscr{B}(\mathscr{H}) \backslash \mathscr{R}^{\prime} \mathscr{S}$ (respectively, if $B \in \mathscr{R} \backslash \mathscr{S}$ ), then there exists $\nu_{0} \in \Omega$ such that $P^{\perp} B Q^{\perp} \notin P^{\perp} \mathscr{R}^{\prime} \mathscr{S} Q^{\perp}$ (respectively, $B \notin P^{\perp} \mathscr{S} Q^{\perp}$ ) for all projections $P, Q$ in $\nu_{0} \cap \mathscr{R}$.

Proof. Choose a basis $\left\{A_{1}, \ldots, A_{r}\right\}$ for $\mathscr{S}$, denote by $\mathscr{R}^{\prime} \odot \mathscr{R}$ the subalgebra of $\mathscr{B}(\mathscr{H} \otimes \mathscr{H})$ generated algebraically by all operators of the form $T^{\prime} \otimes T$, where $T \in \mathscr{R}$ and $T^{\prime} \in \mathscr{R}^{\prime}$, and consider the maps $\varphi: \mathscr{R}^{\prime r} \rightarrow \mathscr{B}(\mathscr{H})$ and $\psi: \mathscr{R}^{\prime r} \rightarrow$ $\mathscr{B}(\mathscr{H} \otimes \mathscr{H})$ defined by

$$
\varphi\left(T_{1}^{\prime}, \ldots, T_{r}^{\prime}\right)=\sum_{j=1}^{r} T_{j}^{\prime} A_{j}
$$

and

$$
\psi\left(T_{1}^{\prime}, \ldots, T_{r}^{\prime}\right)=\sum_{j=1}^{r} T_{j}^{\prime} \otimes A_{j} .
$$

Obviously the range of $\varphi$ is $\mathscr{R}^{\prime} \mathscr{S}$. We regard $\mathscr{R}^{\prime r}$ as a Banach space equipped with the norm $\left\|\left(T_{1}^{\prime}, \ldots, T_{r}^{\prime}\right)\right\|=\max \left\{\left\|T_{j}^{\prime}\right\|: j=1, \ldots, r\right\}$. At the same time $\mathscr{R}^{\prime r}$ is the dual of the Banach space $\mathscr{R}_{\#} \oplus_{1} \cdots \oplus_{1} \mathscr{R}_{\#}$, where $\mathscr{R}_{\#}$ is the predual of 
$\mathscr{R}$, and therefore $\mathscr{R}^{\prime r}$ carries the weak* topology. Recall also that the weak* and the ultraweak topology on $\mathscr{B}(\mathscr{H})$ are the same. It is easy to verify that the maps $\varphi$ and $\psi$ are weak* continuous. Since $\varphi$ is weak* continuous, it is the adjoint of a certain map between the preduals, hence the range of $\varphi$ is weak* closed if and only if it is norm closed [7, p. 173]. Thus, to prove that the range of $\varphi$ is weak* closed it suffices to prove that $\varphi$ is bounded below. To prove this last property of $\varphi$, observe first that $\varphi=\vartheta \psi$, where $\vartheta: R^{\prime} \odot \mathscr{R} \rightarrow \mathscr{B}(\mathscr{H})$ is defined by $\vartheta\left(T_{1}^{\prime} \otimes T_{1}+\cdots+T_{n}^{\prime} \otimes T_{n}\right)=T_{1}^{\prime} T_{1}+\cdots+T_{n}^{\prime} T_{n}$. It is well known that $\vartheta$ is one-to-one and that consequently a $C^{*}$-norm can be defined on $\mathscr{R}^{\prime} \odot \mathscr{R}$ by $\|T\|^{\prime}=\|\vartheta(T)\|$. Since the usual operator norm is minimal among all $C^{*}$-norms on $\mathscr{R}^{\prime} \odot \mathscr{R} \subseteq \mathscr{B}(\mathscr{H} \otimes \mathscr{H})[14$, p. 858], it follows that $\vartheta$ is bounded below by 1 . Thus it suffices now to prove that $\psi$ is bounded below.

Since $A_{1}, \ldots, A_{r}$ are linearly independent, there exist weak-operator continuous linear functionals $\omega_{i}$ on $\mathscr{B}(\mathscr{H})$ with norm 1 such that

$$
\omega_{i}\left(A_{i}\right) \neq 0 \text { and } \omega_{i}\left(A_{j}\right)=0 \text { for } j \neq i, i, j=1, \ldots, r .
$$

For any $\mathbf{T}^{\prime}=\left(T_{1}^{\prime}, \ldots, T_{r}^{\prime}\right) \in \mathscr{R}^{\prime r}$ and any weak-operator continuous linear functional $\rho$ with norm 1 we now have

$$
\left\|\psi\left(\mathbf{T}^{\prime}\right)\right\| \geq\left|\left(\rho \otimes \omega_{i}\right)\left(\psi\left(\mathbf{T}^{\prime}\right)\right)\right|=\left|\sum_{j=1}^{r} \rho\left(T_{j}^{\prime}\right) \omega_{i}\left(A_{j}\right)\right|=\left|\rho\left(T_{i}^{\prime}\right)\right|\left|\omega_{i}\left(A_{i}\right)\right| ;
$$

hence $\left\|\psi\left(\mathbf{T}^{\prime}\right)\right\| \geq c_{i}\left|\rho\left(T_{i}^{\prime}\right)\right|$, where $c_{i}=\left|\omega_{i}\left(A_{i}\right)\right|$. Taking the supremum over all functionals $\rho$ with norm 1 in this inequality we obtain $\left\|\psi\left(\mathbf{T}^{\prime}\right)\right\| \geq c_{i}\left\|T_{i}^{\prime}\right\|$ for each $i=1, \ldots, r$, hence $\left\|\psi\left(\mathbf{T}^{\prime}\right)\right\| \geq c\left\|\mathbf{T}^{\prime}\right\|$, where $c=\min \left\{c_{1}, \ldots, c_{r}\right\}$. This proves that $\psi$ is bounded below.

Since $\varphi$ is weak* continuous and bounded below, $\varphi$ is a weak* homeomorphism from $\mathscr{R}^{\prime r}$ onto the range of $\varphi$. (Indeed, $\varphi$ considered as a map from $\mathscr{R}^{\prime r}$ onto $\operatorname{Im} \varphi$ is an adjoint of a certain map $\varphi_{\#}$ between the preduals, and $\varphi_{\#}$ is one-to-one with dense range, since $\varphi$ is bijective. But, since the range of a linear map between two Banach spaces is closed if and only if the range of its adjoint is closed [7, p. 173], $\varphi_{\#}$ is a bijection, hence by the open mapping theorem $\varphi_{\#}$ is invertible. Then $\varphi^{-1}$ is the adjoint of $\varphi_{\#}^{-1}$, hence $\varphi^{-1}$ is weak* continuous.) If $\mathscr{D}^{\prime}$ is a weak* dense subset of $\mathscr{R}^{\prime}$, then $\mathscr{D}^{\prime r}$ is weak* dense in $\mathscr{R}^{\prime r}$ and $\varphi\left(\mathscr{D}^{\prime r}\right)$ is weak dense in the range of $\varphi$, that is, in $\mathscr{R}^{\prime} \mathscr{S}$. From the obvious fact $\varphi\left(\mathscr{D}^{\prime r}\right) \subseteq \mathscr{D}^{\prime} \mathscr{S}$ it now follows that $\mathscr{D}^{\prime} \mathscr{S}$ must be weak* dense in $\mathscr{R}^{\prime} \mathscr{S}$.

To prove (ii), suppose on the contrary, that for each $\nu \in \Omega$ there exist projections $P_{\nu}, Q_{\nu} \in \nu \cap \mathscr{R}$ such that $P_{\nu}^{\perp} B Q_{\nu}^{\perp} \in P_{\nu}^{\perp} \mathscr{R}^{\prime} \mathscr{S} Q_{\nu}^{\perp}$. Then

$$
P_{\nu}^{\perp} B Q_{\nu}^{\perp}=\sum_{j=1}^{r} T_{j \nu}^{\prime} P_{\nu}^{\perp} A_{j} Q_{\nu}^{\perp}
$$

for suitable $T_{j \nu}^{\prime} \in \mathscr{R}^{\prime}$. For each continuous linear functional $\rho$ with norm 1 
and $i=1, \ldots, r$ we then have

$$
\begin{aligned}
\|B\| & \geq\left\|P_{\nu}^{\perp} B Q_{\nu}^{\perp}\right\| \geq\left\|\sum_{j=1}^{r} T_{j \nu}^{\prime} \otimes P_{\nu}^{\perp} A_{j} Q_{\nu}^{\perp}\right\| \\
& \geq\left|\left(\rho \otimes \omega_{i}\right)\left(\sum_{j=1}^{r} T_{j \nu}^{\prime} \otimes P_{\nu}^{\perp} A_{j} Q_{\nu}^{\perp}\right)\right| \\
& =\left|\sum_{j=1}^{r} \rho\left(T_{j \nu}^{\prime}\right) \omega_{i}\left(P_{\nu}^{\perp} A_{j} Q_{\nu}^{\perp}\right)\right| .
\end{aligned}
$$

Put

$$
d_{i \nu}=\sum_{j=1}^{r} \omega_{i}\left(P_{\nu}^{\perp} A_{j} Q_{\nu}^{\perp}\right) \rho\left(T_{j \nu}^{\prime}\right) \quad \text { for } i=1, \ldots, r,
$$

and consider this as a system of linear equations with unknowns $\rho\left(T_{j \nu}^{\prime}\right)$. Then the solutions can be expressed as

$$
\rho\left(T_{j \nu}^{\prime}\right)=\frac{1}{g_{\nu}} \sum_{i=1}^{r}(-1)^{i+j} g_{i j \nu} d_{i \nu},
$$

where $g_{\nu}=\operatorname{det}\left[\omega_{i}\left(P_{\nu}^{\perp} A_{j} Q_{\nu}^{\perp}\right)\right]$ and $g_{i j \nu}$ are certain subdeterminants of $g_{\nu}$ of dimension $(r-1) \times(r-1)$. With $a=\max \left\{\left\|A_{j}\right\|: j=1, \ldots, r\right\}$, we obviously have $\left|g_{i j \nu}\right| \leq(r-1) ! a^{r-1}$ (since $\omega_{i}, P_{\nu}^{\perp}$, and $Q_{\nu}^{\perp}$ have norms 1). Since $\left|d_{i \nu}\right| \leq\|B\|$ (by (3.2)), it follows that

$$
\left|\rho\left(T_{j \nu}^{\prime}\right)\right| \leq r ! a^{r-1}\|B\|\left|g_{\nu}\right|^{-1} \text { for all } j=1, \ldots, r .
$$

Since the net $\left\{P_{\nu}^{\perp} A_{j} Q_{\nu}^{\perp}: \nu \in \Omega\right\}$ converges strongly to $A_{j}$ for each $j$ and since the functionals $\omega_{i}$ are strongly continuous, it follows that the net $\left\{g_{\nu}: \nu \in \Omega\right\}$ converges to $\operatorname{det}\left[\omega_{i}\left(A_{j}\right)\right]=\omega_{1}\left(A_{1}\right) \times \cdots \times \omega_{r}\left(A_{r}\right) \neq 0$. By (3.3) this implies that there exists $\nu_{0} \in \Omega$ such that the set $\left\{\left|\rho\left(T_{j \nu}^{\prime}\right)\right|: \nu \subseteq \nu_{0}, \nu \in \Omega\right\}$ is bounded for each $j=1, \ldots, r$, and the bound is independent of $\rho$. Taking the supremum over all $\rho$ with norm 1 , we see that the sets $\left\{\left\|T_{j \nu}^{\prime}\right\|: \nu \subseteq \nu_{0}\right\}$ are bounded. By the weak-operator compactness of balls in $\mathscr{R}^{\prime}$, there exist subnets $\left\{T_{j \eta}^{\prime}\right\}$ of $\left\{T_{j \nu}^{\prime}\right\}$ such that $T_{j \eta}^{\prime} \rightarrow T_{j}^{\prime}$, where $T_{j}^{\prime} \in \mathscr{R}^{\prime}$. Taking the weak-operator limit in (3.1) along these subnets we obtain $B=\sum_{j=1}^{r} T_{j}^{\prime} A_{j} \in \mathscr{R}^{\prime} \mathscr{S}$ (since $P_{\eta}^{\perp} A_{j} Q_{\eta}^{\perp} \rightarrow A_{j}$ strongly, $T_{j \eta}^{\prime} \rightarrow T_{j}^{\prime}$ weakly, and $\left\{T_{j \eta}^{\prime}\right\}$ is bounded for each $\left.j\right\}$, but this is in contradiction with the hypothesis that $B \notin \mathscr{R}^{\prime} \mathscr{S}$.

We have to prove also that for $B \in \mathscr{R} \backslash \mathscr{S}$ there exists $\nu_{0} \in \Omega$ such that $P^{\perp} B Q^{\perp} \notin P^{\perp} \mathscr{S} Q^{\perp}$ for all projections $P, Q \in \nu_{0} \cap \mathscr{R}$, but this follows easily from the linear independence of the set $\left\{B, A_{1}, \ldots, A_{r}\right\}$ and the compactness of the unit sphere in $\mathbb{C}^{r+1}$ by arguments similar (but much simpler) to those which we have already used above. 
Now we are going to show that Theorem 1.3 for factors follows from the following technical lemma.

Lemma 3.2. Let $\mathscr{R}$ be a von Neumann algebra on $\mathscr{H}$ without nonzero minimal projections. If $\left\{A_{1}, \ldots, A_{r}\right\}$ is any subset of $\mathscr{R}$ and $C, D$ are projections in $\mathscr{R}$ such that $C A_{r} D \neq 0$, then for each neighborhood $\nu \in \Omega$ there exists subprojections $E \leq C$ and $F \leq D$ such that $E A_{r} F \neq 0$ and $P, Q \in \nu$, where

$$
P=E \vee \bigvee_{j=1}^{r} R\left(A_{j} F\right) \text { and } Q=F \vee \bigvee_{j=1}^{r} R\left(A_{j}^{*} E\right)
$$

Proof of Theorem 1.3 for factors (assuming Lemma 3.2). If $\mathscr{R}$ is of type $\mathrm{I}_{1}$, the proof is trivial, so we assume that $\mathscr{R}$ is a factor of type II or III. The proof is by induction on the dimension of $\mathscr{S}$. By Corollary 2.6 the theorem is true if $\mathscr{S}$ is one-dimensional, and by Lemma 3.1 we then have $\operatorname{ref}\left(\mathscr{R}^{\prime} \mathscr{S}\right)=$ $\mathscr{R}^{\prime} \mathscr{S}$. Assume inductively that $\operatorname{ref}_{\mathscr{R}}(\mathscr{T})=\mathscr{T}$ and $\operatorname{ref}\left(\mathscr{R}^{\prime} \mathscr{T}\right)=\mathscr{R}^{\prime} \mathscr{T}$ for all subspaces $\mathscr{T}$ in $\mathscr{R}$ of dimension smaller than a given integer $r$, let $\mathscr{S}$ be any $r$-dimensional subspace of $\mathscr{R}$, and choose a basis $\left\{A_{1}, \ldots, A_{r}\right\}$ for $\mathscr{S}$. We shall prove here only the identity $\operatorname{ref}\left(\mathscr{R}^{\prime} \mathscr{S}\right)=\mathscr{R}^{\prime} \mathscr{S}$, since the proof of the identity $\operatorname{ref}_{\mathscr{R}}(\mathscr{S})=\mathscr{S}$ is the same. (To prove the second identity, $\mathscr{R}^{\prime}$ must be replaced by $\mathscr{C}=\mathbb{C I}$ in the arguments below, and only the easy part of Lemma 3.1(ii) is needed.) We shall prove that for each $A_{r+1} \in \mathscr{B}(\mathscr{H}) \backslash \mathscr{R}^{\prime} \mathscr{S}$ there exist $X, Y \in \mathscr{R}$ such that

$$
X A_{j} Y=0 \text { for } j=1, \ldots, r \text { and } X A_{r+1} Y \neq 0 .
$$

By Lemma 1.1 this means that $A_{r+1} \notin \operatorname{ref}\left(\mathscr{R}^{\prime} \mathscr{S}\right)$, hence this will complete the proof.

By the inductive hypothesis (applied to the span of $\left.\left\{A_{1}, \ldots, A_{r-1}\right\}\right)$ there exist projections $C, D \in \mathscr{R}$ such that

$$
C A_{j} D=0 \text { for } j=1, \ldots, r-1 \text { and } C A_{r} D \neq 0 .
$$

If $C A_{r+1} D \notin \mathscr{R}^{\prime} C A_{r} D$, then by Corollary 2.6 and Lemma 3.1 there exist $S, T \in \mathscr{R}$ such that $S\left(C A_{r} D\right) T=0$ and $S\left(C A_{r+1} D\right) T \neq 0$, hence we obtain the desired relations $(*)$ by putting $X=S C$ and $Y=D T$.

We may therefore assume that $C A_{r+1} D=S^{\prime} C A_{r} D$ for some $S^{\prime} \in \mathscr{R}^{\prime}$. Since $A_{r+1} \notin \mathscr{R}^{\prime} \mathscr{S}$, by Lemma 3.1 there exists a neighborhood $\nu \in \Omega$ such that $P^{\perp} A_{r+1} Q^{\perp} \notin \mathscr{R}^{\prime} P^{\perp} \mathscr{S} Q^{\perp}$ for each projections $P, Q \in \nu \cap \mathscr{R}$. By Lemma 3.2 there exist projections $E \leq C$ and $F \leq D$ in $\mathscr{R}$ such that $E A_{r} F \neq 0$ and $P, Q \in \nu$, where

$$
P=E \vee \bigvee_{j=1}^{r+1} R\left(A_{j} F\right) \text { and } Q=F \vee \bigvee_{j=1}^{r+1} R\left(A_{j}^{*} E\right)
$$

Since $E \leq C$ and $F \leq D$, we now have

$$
E A_{j} F=0 \text { for } j=1, \ldots, r-1, \quad E A_{r+1} F \neq 0,
$$


and (from $C A_{r+1} D=S^{\prime} C A_{r} D$ )

$$
E A_{r+1} F=S^{\prime} E A_{r} F .
$$

From $P^{\perp} A_{r+1} Q \notin \mathscr{R}^{\prime} P^{\perp} \mathscr{S} Q^{\perp}$ it follows

$$
P^{\perp}\left(A_{r+1}-S^{\prime} A_{r}\right) Q^{\perp} \notin \sum_{j=1}^{r-1} \mathscr{R}^{\prime} P^{\perp} A_{j} Q^{\perp},
$$

hence by the inductive hypothesis there exist projections $G, H \in \mathscr{R}$ such that $G P^{\perp} A_{j} Q^{\perp} H=0$ for $j=1, \ldots, r-1$ and $G P^{\perp}\left(A_{r+1}-S^{\prime} A_{r}\right) Q^{\perp} H \neq 0$. We may assume that $G \leq P^{\perp}$ and $H \leq Q^{\perp}$ (otherwise we just replace $G$ by $N\left(G P^{\perp}\right)^{\perp}$ and $H$ by $\left.R\left(Q^{\perp} H\right)\right)$, hence we have

$$
G A_{j} H=0 \text { for } j=1, \ldots, r-1
$$

and

$$
G\left(A_{r+1}-S^{\prime} A_{r}\right) H \neq 0 .
$$

Now put $K=E+G$ and $L=F+H$. Then $K$ and $L$ are projections (since $E \leq P, G \leq P^{\perp}, F \leq Q$, and $\left.H \leq Q^{\perp}\right)$. From the definition of $P$ and $Q$ we see that $R\left(A_{j} F\right) \leq P$ and $Q^{\perp} \leq N\left(E A_{j}\right)$ for all $j=1, \ldots, r+1$. Since $G \leq P^{\perp}$ and $H \leq Q^{\perp}$, this implies $G A_{j} F=0$ and $E A_{j} H=0$, thus we have

$$
\begin{aligned}
K A_{j} L & =(E+G) A_{j}(F+H) \\
& =E A_{j} F+G A_{j} H \text { for all } j=1, \ldots, r+1 .
\end{aligned}
$$

From (3.8), (3.4), and (3.6) we obtain

$$
K A_{j} L=0 \text { for } j=1, \ldots, r-1 .
$$

Moreover, we claim that $K A_{r+1} L \notin \mathscr{R}^{\prime} K A_{r} L$. To prove this, suppose on the contrary that $K A_{r+1} L=T^{\prime} K A_{r} L$ for some $T^{\prime} \in \mathscr{R}^{\prime}$. From (3.8) and (3.5) we then obtain

$$
S^{\prime} E A_{r} F+G A_{r+1} H=T^{\prime} E A_{r} F+T^{\prime} G A_{r} H .
$$

Since $E \perp G$, this implies $S^{\prime} E A_{r} F=T^{\prime} E A_{r} F$, or $\left(T^{\prime}-S^{\prime}\right) E A_{r} F=0$. Since $T^{\prime}-S^{\prime} \in \mathscr{R}^{\prime}, E A_{r} F \in \mathscr{R}, E A_{r} F \neq 0$, and $\mathscr{R}$ is a factor, the last equality implies $T^{\prime}-S^{\prime}=0$. Putting now $T^{\prime}=S^{\prime}$ in (3.10) we obtain $G A_{r+1} H=$ $S^{\prime} G A_{r} H$, but this is in contradiction with (3.7).

Since $K A_{r+1} L \notin \mathscr{R}^{\prime} K A_{r} L$, by Corollary 2.6 (together with Lemma 3.1 and Lemma 1.1) there exist two operators $V, W \in \mathscr{R}$ such that $V\left(K A_{r} L\right) W=0$ and $V\left(K A_{r+1} L\right) W \neq 0$. Thus, denoting $X=V K$ and $Y=L V$ and recalling (3.9), we obtain the desired relations $(*)$.

If $\mathscr{R}$ is a factor of type I and $\mathscr{S}$ is a finite-dimensional subspace of $\mathscr{R}$ which has zero intersection with the ideal $\mathscr{F}$ generated by finite projections in 
$\mathscr{R}$, the above proof can easily be modified to show that $\mathscr{S}$ is $\mathscr{R}$-reflexive, and Lemma 3.2 is not needed in this case.

The proof of Lemma 3.2 is very short if $\mathscr{R}$ is a factor of type II. Namely, in this case we may assume that the projections $C$ and $D$ in Lemma 3.2 are finite (otherwise we just replace them with suitable finite subprojections such that the condition $C A_{r} D \neq 0$ is still satisfied). Moreover, since each projection in $\mathscr{R}$ can be expressed as an orthogonal sum of two equivalent subprojections [14, $\mathrm{p}$. 426], we can find two decreasing sequences, $\left(E_{n}\right)$ and $\left(F_{n}\right)$, of subprojections of $C$ and $D$, respectively, such that $E_{n} A_{r} F_{n} \neq 0$ for each $n$ and $\rho\left(E_{n}\right) \leq 2^{-n}$, $\rho\left(F_{n}\right) \leq 2^{-n}$, where $\rho$ is a faithful normal semifinite tracial weight on $\mathscr{R}$ $[14$, p. 541]. Using the fact that the range projection of each operator $T$ is equivalent to the range projection of $T^{*}$ and Kaplansky's formula [14, p. 403], we now have

$$
\begin{aligned}
\rho\left(E_{n} \vee \bigvee_{j=1}^{r} R\left(A_{j} F_{n}\right)\right) & \leq \rho\left(E_{n}\right)+\sum_{j=1}^{r} \rho\left(R\left(A_{j} F_{n}\right)\right) \\
& =\rho\left(E_{n}\right)+\sum_{j=1}^{r} \rho\left(R\left(F_{n} A_{j}^{*}\right)\right) \leq(r+1) 2^{-n} .
\end{aligned}
$$

Since the sequences $\left(E_{n}\right)$ and $\left(F_{n}\right)$ are decreasing and $\rho$ is faithful, it follows that the projections

$$
P_{n}=E_{n} \vee \bigvee_{j=1}^{r} R\left(A_{j} F_{n}\right) \quad\left(\text { and similarly } Q_{n}=F_{n} \vee \bigvee_{j=1}^{r} R\left(A_{j}^{*} E_{n}\right)\right)
$$

converge to 0 . This proves Lemma 3.2 for factors of type II.

The proof of Lemma 3.2 for general von Neumann algebras without nonzero minimal projections is a little more technical, although elementary. The lemma is needed only for factors, but the assumption that $\mathscr{R}$ is a factor of type III would not simplify the proof. We need three additional lemmas, the first two of which are very short.

Lemma 3.3. If $E, F \in \mathscr{B}(\mathscr{H})$ are two projections such that $\|F E\|<1$, then $E \vee F \leq a(E+F)$, where $a=(1-\|E F\|)^{-1}$.

Proof. It is well known that $E \vee F=R(E+F)$ [9]. For each $x \in E \mathscr{H}$ and $y \in F \mathscr{H}$ we have

$$
\begin{aligned}
\|x+y\|^{2} & \geq\|x\|^{2}+\|y\|^{2}-2|\langle x, y\rangle| \geq\|x\|^{2}+\|y\|^{2}-2\|E F\|\|x\|\|y\| \\
& \geq(1-\|E F\|)\left(\|x\|^{2}+\|y\|^{2}\right),
\end{aligned}
$$

or $\|x\|^{2}+\|y\|^{2} \leq a\|x+y\|^{2}$. Putting $x=E z$ and $y=F z$ in this inequality, where $z \in \mathscr{H}$ is arbitrary, we obtain

$$
\langle(E+F) z, z\rangle=\|E z\|^{2}+\|F z\|^{2} \leq a\|(E+F) z\|^{2}=a\left\langle(E+F)^{2} z, z\right\rangle .
$$

This means that $E+F \leq a(E+F)^{2}$, hence (by functional calculus) $R(E+F) \leq$ $a(E+F)$. 
Lemma 3.4. Let $\mathscr{R}$ be any von Neumann algebra on $\mathscr{H}, A \in \mathscr{R}$, and $F \in$ $\operatorname{Proj}(\mathscr{R})$. If $A \mathscr{H} \nsubseteq F \mathscr{H}$, then there exists a subprojection $G \leq N(A)^{\perp}$ in $\mathscr{R}$ such that $G \neq 0$ and $\|F R(A G)\|<1$.

Proof. From $A \mathscr{H} \nsubseteq F \mathscr{H}$ we have $A^{*} F^{\perp} A \neq 0$, hence $A^{*} A-A^{*} F A>0$. Thus, there exists $t \in(0,1)$ such that the positive part of the operator $S=$ $t A^{*} A-A^{*} F A$ is nonzero. Let $G$ be the spectral projection of $S$ corresponding to the strictly positive part of the spectrum of $S$. Then $G \perp N(S)$ and it follows that $G \leq N(A)^{\perp}$, since $\operatorname{Ker} A \subseteq \operatorname{Ker} S$. Further, from $G S G \geq 0$ we have $G A^{*} F A G \leq t G A^{*} A G$, or $\|F A G x\|^{2} \leq t\|A G x\|^{2}$ for all $x \in \mathscr{H}$. This implies $\|F \mid A G \mathscr{H}\| \leq t^{1 / 2}$, which is clearly equivalent to $\|F R(A G)\| \leq t^{1 / 2}$.

Lemma 3.5. Let $\mathscr{R}$ be a von Neumann algebra on $\mathscr{H}$ without nonzero minimal projections. Then:

(i) For each finite subset $\left\{A_{1}, \ldots, A_{r}\right\}$ of $\mathscr{R}$, each nonzero projection $E \in$ $\mathscr{R}$, and each strong neighborhood $\nu_{0}$ of 0 there exists a nonzero subprojection $E_{0}$ of $E$ in $\mathscr{R}$ such that the projection $\bigvee_{j=1}^{r} R\left(A_{j} E_{0}\right)$ is in $\nu_{0} \cap \mathscr{R}$.

(ii) For all projections $E, F$ in $\mathscr{R}$, where $E \neq 0$, and for each strong neighborhood $\nu_{0}$ of 0 there exists a nonzero subprojection $E_{0}$ of $E$ in $\mathscr{R}$ such that $F \vee E_{0} \in F+\nu_{0}$.

Proof. (i) The proof is by induction on $r$. Suppose first that $r=1$, denote $A_{1}$ simply by $A$, and let $A E=U|A E|$ be the usual polar decomposition of $A E$. We may assume that $A E \neq 0$, otherwise the proof is trivial. There exists an orthogonal sequence of nonzero projections $G_{n}$ in $\mathscr{R}$ such that $G_{n} \leq$ $R(|A E|)$ and $G_{n}$ reduces $|A E|$ for each $n$. Indeed, if the spectrum of $|A E|$ is infinite, then we can obviously take for $G_{n}$ suitable spectral projections. If, on the other hand, the spectrum of $|A E|$ is finite, then let $G$ be the spectral projection of $|A E|$ corresponding to some nonzero eigenvalue of $|A E|$ and let $\left(G_{n}\right)$ be any orthogonal sequence of nonzero subprojections of $G$ in $\mathscr{R}$. (Such a sequence exists, since $\mathscr{R}$ does not contain nonzero minimal projections.) Since $G_{n} \leq R(|A E|) \leq E$ and since $U$ is isometric on $[|A E| \mathscr{H}]$, we now have $\left[A G_{n} \mathscr{H}\right]=\left[A E G_{n} \mathscr{H}\right]=U\left[|A E| G_{n} \mathscr{H}\right]=U G_{n} \mathscr{H}=U G_{n} U^{*} \mathscr{H}$, hence $R\left(A G_{n}\right)=U G_{n} U^{*}$. This implies in particular that the sequence $\left(R\left(A G_{n}\right)\right)$ converges strongly to 0 , hence $G_{n} \in \nu_{0}$ for all sufficiently large $n$ and we may put $E_{0}=G_{n}$ for one such $n$.

Suppose now inductively that part (i) of the lemma holds for all neighborhoods $\nu$, all projections $E \in \mathscr{R}$, and all subsets of $\mathscr{R}$ which have at most $r-1$ elements, where $r$ is a fixed positive integer. By the inductive hypothesis (applied to the set $\left\{A_{1}, \ldots, A_{r-1}\right\}$, to projection $E$, and to the given neighborhood $\nu_{0} \in \Omega$ ) there exists a nonzero subprojection $G_{0}$ of $E$ in $\mathscr{R}$ such that $P_{0} \in \nu_{0}$, where

$$
P_{0}=\bigvee_{j=1}^{r-1} R\left(A_{j} G_{0}\right) .
$$

If $P_{0} \vee R\left(A_{r} G_{0}\right) \in \nu_{0}$, then the proof is completed by putting $E_{0}=G_{0}$. So 
assume that $P_{0} \vee R\left(A_{r} G_{0}\right) \notin \nu_{0}$. Then $\left(A_{r} G_{0}\right) \mathscr{H} \nsubseteq P_{0} \mathscr{H}$ (since $\left.P_{0} \in \nu_{0}\right)$, hence by Lemma 3.4 there exists a nonzero subprojection $H \leq N\left(A_{r} G_{0}\right)^{\perp} \leq G_{0}$ in $\mathscr{R}$ such that

$$
b=\left\|P_{0} R\left(A_{r} H\right)\right\|<1 .
$$

By the already proved case $r=1$ (applied to the operator $A_{r}$ and projection $H$ ) there exists for each $\nu \in \Omega$ a nonzero subprojection $K_{\nu}$ of $H$ in $\mathscr{R}$ such that $R\left(A_{r} K_{\nu}\right) \in \nu$. By the inductive hypothesis (applied to the set $\left\{A_{1}, \ldots, A_{r-1}\right\}$ and projection $\left.K_{\nu}\right)$ there exists for each $\nu \in \Omega$ a nonzero subprojection $L_{\nu}$ of $K_{\nu}$ in $\mathscr{R}$ such that $Q_{\nu} \in \nu$, where

$$
Q_{\nu}=\bigvee_{j=1}^{r-1} R\left(A_{j} L_{\nu}\right)
$$

From $L_{\nu} \leq K_{\nu} \leq H \leq G_{0}$ we now have $R\left(A_{r} L_{\nu}\right) \leq R\left(A_{r} H\right)$ and $Q_{\nu} \leq P_{0}$, hence (3.11) implies that $\left\|Q_{\nu} R\left(A_{r} L_{\nu}\right)\right\| \leq b<1$ for all $\nu \in \Omega$. By Lemma 3.3 we now have

$$
Q_{\nu} \vee R\left(A_{r} L_{\nu}\right) \leq a\left(Q_{\nu}+R\left(A_{r} L_{\nu}\right)\right) \text { for all } \nu \in \Omega,
$$

where $a=(1-b)^{-1}$. Since $Q_{\nu} \in \nu$ and $R\left(A_{r} L_{\nu}\right) \leq R\left(A_{r} K_{\nu}\right) \in \nu$ for all $\nu \in \Omega$, we see from (3.12) that the net $\left\{Q_{\nu} \vee R\left(A_{r} L_{\nu}\right): \nu \in \Omega\right\}$ converges strongly to 0 . It follows that for the given neighborhood $\nu_{0}$ there exists $\nu \in \Omega$ such that $Q_{\nu} \vee R\left(A_{r} L_{\nu}\right) \in \nu_{0}$ and this proves part (i) of the lemma (put $E_{0}=L_{\nu}$ ).

(ii) For each $\nu \in \Omega$ let $E_{\nu} \in \mathscr{R} \cap \nu$ be a nonzero subprojection of $E$ (here again the fact that $\mathscr{R}$ has no nonzero minimal projections is used). If $E_{\nu_{0}} \leq F$, then, denoting $E_{0}=E_{\nu_{0}}$, we have $E_{0} \vee F=F \in F+\nu_{0}$ and the proof is completed in this case. Assume therefore that $E_{\nu_{0}} \mathscr{H} \nsubseteq F \mathscr{H}$. Then by Lemma 3.4 there exists a nonzero subprojection $G$ of $E_{\nu_{0}}$ in $\mathscr{R}$ such that $\|F G\|<1$. Since $\mathscr{R}$ has no nonzero minimal projections, there exists a decreasing sequence $\left(G_{n}\right)$ of nonzero subprojections of $G$ in $\mathscr{R}$ that converges strongly to 0 . Then of course $\left\|F G_{n}\right\| \leq\|F G\|$ and Lemma 3.3 implies that $F \vee G_{n} \leq a\left(F+G_{n}\right)$, where $a=(1-\|F G\|)^{-1}$. The decreasing sequence $\left(F \vee G_{n}\right)$ has the strong operator limit, say $H$, and from $F \leq F \vee G_{n} \leq a\left(F+G_{n}\right)$ we have $F \leq H \leq a F$ (since $G_{n} \rightarrow 0$ ). Since $F$ and $H$ are projections, this implies $H=F$. So the sequence $\left(F \vee G_{n}\right)$ converges to $F$ and, consequently, $F \vee G_{n} \in F+\nu_{0}$ for all large enough $n$; put $E_{0}=G_{n}$ for one such $n$.

Proof of Lemma 3.2. We may assume that $C A_{r} \widetilde{D} \neq 0$ for all nonzero subprojections $\widetilde{D}$ of $D$ in $\mathscr{R}$ (otherwise replace $D$ by $D-\left(N\left(C A_{r}\right) \wedge D\right)$ ). By Lemma 3.5(i) there exists for each neighborhood $\nu \in \Omega$ a nonzero subprojection $D_{0}$ of $D$ in $\mathscr{R}$ such that $P_{0} \in \frac{1}{2} \nu$, where

$$
P_{0}=\bigvee_{j=1}^{r} R\left(A_{j} D_{0}\right)
$$


Replacing $C$ by the smaller projection $C-\left(C \wedge R\left(A_{r} D_{0}\right)^{\perp}\right)$, we may assume that $\widetilde{C} A_{r} D_{0} \neq 0$ for all nonzero subprojections $\widetilde{C}$ of $C$ in $\mathscr{R}$. By Lemma 3.5(ii) (applied to projections $C$ and $P_{0}$ ) there exists a nonzero subprojection $C_{0}$ of $C$ in $\mathscr{R}$ such that $P_{0} \vee C_{0} \in P_{0}+\frac{1}{2} \nu$, hence we now have $C_{0} A_{r} D_{0} \neq 0$ and $P_{0} \vee C_{0} \in \nu$ (since $\left.P_{0} \in \frac{1}{2} \nu\right)$, or

$$
C_{0} \vee \bigvee_{j=1}^{r} R\left(A_{j} D_{0}\right) \in \nu
$$

The projections $C_{0}$ and $D_{0}$ still do not necessarily satisfy all the requirements of Lemma 3.2, since we cannot be sure that the projection

$$
D_{0} \vee \bigvee_{j=1}^{r} R\left(A_{j}^{*} C_{0}\right)
$$

is in $\nu$. But repeating the above arguments with $\left\{A_{1}^{*}, \ldots, A_{r}^{*}\right\}, D_{0}$, and $C_{0}$ in place of $\left\{A_{1}, \ldots, A_{r}\right\}, C$, and $D$, respectively, we obviously obtain subprojections $E$ and $F$ of $C$ and $D$ (respectively) that satisfy all the requirements of Lemma 3.2.

Since each reflexive subspace of $\mathscr{B}(\mathscr{H})$ is obviously closed in the weak operator topology, we have the following

Corollary 3.6. If $\mathscr{S}$ is a finite-dimensional subspace of any factor $\mathscr{R}$ on $\mathscr{H}$, then the space $\mathscr{R}^{\prime} \mathscr{S}$ is closed in the weak operator topology.

Proof. If $\mathscr{R}$ is of type II or III, the corollary follows immediately from the already proved case of Theorem 1.3 for factors together with Lemma 3.1. If $\mathscr{R}$ is of type $\mathrm{I}$, then there exists a Hilbert space $\mathscr{H}$ and a cardinal $n$ such that $\mathscr{R}$ is (unitarily equivalent to) $\mathscr{B}(\mathscr{K})^{(n)}$; hence $\mathscr{R}^{\prime}=M_{n}\left(\mathbb{C I}_{\mathscr{K}}\right)$ and there exists a subspace $\mathscr{T}$ of $\mathscr{B}(\mathscr{K})$ such that $\mathscr{S}=\mathscr{T}^{(n)}$. (In the language of tensor products this means that there exist Hilbert spaces $\mathscr{K}$ and $\mathscr{G}$ such that $\mathscr{R}$ is (unitarily equivalent to) $\mathscr{B}(\mathscr{K}) \otimes \mathbb{C} I_{\mathscr{G}}$ and $\mathscr{R}^{\prime}=\mathbb{C}_{\mathscr{K}} \otimes \mathscr{B}(\mathscr{G})[14$, p. 815].) Since $\mathscr{S}$ is finite-dimensional, it is now easy to see that $\mathscr{R}^{\prime} \mathscr{S}=M_{n}(\mathscr{T})$ (that is, $\mathscr{R}^{\prime} \mathscr{S}$ consists of bounded matrices with entries in $\mathscr{T}$ ). This implies that $\mathscr{R}^{\prime} \mathscr{S}$ is closed in the weak operator topology.

Remark. For a countably decomposable ( $=\sigma$-finite) factor $\mathscr{R}$ of type III part (ii) of Theorem 1.3 can be improved in the following way: if $\mathscr{S}$ is a finitedimensional subspace of $\mathscr{R}$ and $\varphi: \mathscr{S} \rightarrow \mathscr{R}$ is any linear map, then there exist $X, Y$ in $\mathscr{R}$ such that $X A Y=\varphi(A)$ for each $A \in \mathscr{S}$. (A similar result is proved in [21] for the Calkin algebra.) The proof uses Theorem 1.3(ii), but it is quite technical and will not be given here. We merely remark that such a result is possible only in simple algebras ( = without two-sided ideals).

\section{Reflexivity in General von NeumanN algebras}

In this section we shall first complete the proof of Theorem 1.3. The theorem has been already proved for factors in the previous section. Here it will be 
extended first to general von Neumann algebras acting on separable spaces, then a very short argument will show that the theorem holds for all countably generated von Neumann algebras. Although there is no reason why the theorem should not be true for completely general von Neumann algebras, the author has been unable to deduce this in a simple way. It is possible to extend the reduction theory of [6] from non-self-adjoint algebras to subspaces of operators, but since we are dealing here only with special kinds of subspaces, the ordinary selfadjoint reduction theory will be sufficient. We refer to [14 or 27] for complete treatments of reduction theory and to $[6, \S 2]$ for a brief survey of the notions used here. Our first very simple lemma will imply that for any vector space of decomposable operators $\mathscr{T}, \operatorname{ref}(\mathscr{T})$ consists also of decomposable operators.

Lemma 4.1. Let $\mathscr{R}$ be a von Neumann algebra on $\mathscr{H}$ with centre $\mathscr{C}$ and let $\mathscr{T}$ be any subspace of $\mathscr{C}^{\prime}$. Then $\operatorname{ref}(\mathscr{T}) \subseteq \mathscr{C}^{\prime}$.

Proof. Let $B \in \operatorname{ref}(\mathscr{T})$. For any projection $P$ in $\mathscr{C}$ we have $P^{\perp} \mathscr{T} P=0$, hence $P^{\perp} B P=0$. Interchanging the roles of $P$ and $P^{\perp}$, we see that $B$ commutes with $P$, hence (since $P$ is arbitrary) $B \in \mathscr{C}^{\prime}$.

Proof of Theorem 1.3. We shall prove in detail only part (i), since the proof of part (ii) is very similar. Only the inclusion $\operatorname{ref}\left(\mathscr{R}^{\prime} \mathscr{S}\right) \subseteq \overline{\mathscr{R}}^{\prime} \mathscr{S}$ needs a proof, the opposite inclusion is trivial.

Assume first that the Hilbert space $\mathscr{H}$, on which $\mathscr{R}$ acts, is separable. Then there exists the direct integral decompositions of $\mathscr{R}$ and $\mathscr{H}$ along the centre $\mathscr{C}$ of $\mathscr{R}$, so that

$$
\mathscr{H}=\int_{\Lambda}^{\oplus} \mathscr{H}(\lambda) d \mu(\lambda) \text { and } \mathscr{R}=\int_{\Lambda}^{\oplus} \mathscr{R}(\lambda) d \mu(\lambda),
$$

where $\Lambda$ is a complete separable (locally compact) metric space and $\mu$ is a positive complete Borel measure on $\Lambda$. Moreover, the algebra of all diagonal operators coincides with $\mathscr{C}$ and $\mathscr{R}^{\prime}$ can be decomposed as $\mathscr{R}^{\prime}=\int_{\Lambda}^{\oplus} \mathscr{R}^{\prime}(\lambda) d \mu(\lambda)$, where $\mathscr{R}^{\prime}(\lambda)=\mathscr{R}(\lambda)^{\prime}$ almost everywhere. Choose a basis $\left\{A_{1}, \ldots, A_{r}\right\}$ for $\mathscr{S}$ and let $A_{j}=\int_{\Lambda}^{\oplus} A_{j}(\lambda) d \mu(\lambda)$ be the decomposition of $A_{j}$, where $A_{j}()$ are Borel functions on $\Lambda$. For each $\lambda \in \Lambda$ let $\mathscr{S}(\lambda)=\operatorname{span}\left\{A_{1}(\lambda), \ldots, A_{r}(\lambda)\right\}$; clearly another choice of basis and representing functions gives a family of spaces which agrees almost everywhere with $\mathscr{S}(\lambda)$. Let

$$
\mathscr{T}=\left\{T \in \mathscr{C}^{\prime}: T(\lambda) \in \mathscr{R}^{\prime}(\lambda) \mathscr{S}(\lambda) \text { for almost all } \lambda \in \Lambda\right\},
$$

where, of course, $T=\int_{\Lambda}^{\oplus} T(\lambda) d \mu(\lambda)$. We shall prove that

$$
\operatorname{ref}\left(\mathscr{R}^{\prime} \mathscr{S}\right) \subseteq \mathscr{T} \subseteq \overline{\mathscr{R}^{\prime} \mathscr{S}}
$$

this will complete the proof of part (i) of the theorem. For simplicity of notation let us assume that the spaces $\mathscr{H}(\lambda)$ are all equal to a fixed Hilbert space $\mathscr{H}$; the general case is treated in the same way by using measurable transfer $\left[14\right.$, p. 1020]. In this case $\mathscr{H}$ is identified with the Hilbert space $L_{2}(\Lambda, \mathscr{K}, \mu)$ of all equivalence classes of measurable functions $x$ from $\Lambda$ to $\mathscr{K}$ satisfying 
$\int_{\Lambda}\|x(\lambda)\|^{2} d \mu(\lambda)<\infty$, the algebra $\mathscr{C}^{\prime}$ of all decomposable operators consists of all multiplications by measurable mappings $T: \Lambda \rightarrow \mathscr{B}(\mathscr{K})$ such that the function $\lambda \rightarrow\|T(\lambda)\|$ is essentially bounded, and $\mathscr{C}$ consists of all multiplications by functions of the form $\lambda \rightarrow f(\lambda) I$, where $f \in L_{\infty}(\Lambda, \mu)$.

To prove the first inclusion in (4.1) assume on the contrary that there exists $B \in \operatorname{ref}\left(\mathscr{R}^{\prime} \mathscr{S}\right) \backslash \mathscr{T}$. Since $B \in \operatorname{ref}\left(\mathscr{R}^{\prime} \mathscr{S}\right)$ and $\mathscr{R}^{\prime} \mathscr{S} \subseteq \mathscr{C}^{\prime}$, Lemma 4.1 implies that $B$ is decomposable; let $B=\int_{\Lambda}^{\oplus} B(\lambda) d \mu(\lambda)$ be the decomposition of $B$, where $B()$ is a Borel operator valued function. Let $\left\{A_{n}^{\prime}\right\}_{n=1}^{\infty}$ be a countable strongly dense subset of the unit ball of $\mathscr{R}^{\prime}$. By standard theorems of the reduction theory there exists a Borel subset $\Lambda_{0}$ of $\Lambda$ such that $\mu\left(\Lambda \backslash \Lambda_{0}\right)=0$ and for each $\lambda \in \Lambda_{0}$ the following conditions are satisfied: $\mathscr{S}(\lambda) \subseteq \mathscr{R}(\lambda)$ (that is, $A_{j}(\lambda) \in \mathscr{R}(\lambda)$ for $\left.j=1, \ldots, r\right)$, the set $\left\{A_{n}^{\prime}(\lambda)\right\}_{n=1}^{\infty}$ is strongly dense in the unit ball of $\mathscr{R}^{\prime}(\lambda), \mathscr{R}^{\prime}(\lambda)=\mathscr{R}(\lambda)^{\prime}$, and $\mathscr{R}(\lambda)$ is a factor of type $I_{1}$, II, or III. (For the last condition, the assumption that $\mathscr{R}$ has no central portions of type $I_{n}$ for $n>1$ has been used.) Consider the subset $Z$ of $\Lambda_{0} \times(\operatorname{Proj}(\mathscr{K}) \times \operatorname{Proj}(\mathscr{K}))$ consisting of all triples $(\lambda ; P, Q)$ satisfying the following conditions:

(i) $P A_{n}^{\prime}(\lambda)=A_{n}^{\prime}(\lambda) P$ and $Q A_{n}^{\prime}(\lambda)=A_{n}^{\prime}(\lambda) Q$ for all $n=1,2, \ldots$;

(ii) $P A_{j}(\lambda) Q=0$ for all $j=1, \ldots, r$;

(iii) $P B(\lambda) Q \neq 0$.

Standard arguments show that $Z$ is a Borel (hence analytic) subset of the Polish space $\Lambda \times \operatorname{Proj}(\mathscr{K}) \times \operatorname{Proj}(\mathscr{K})$, where $\operatorname{Proj}(\mathscr{K})$ is of course equipped with the strong operator topology. Denote by $\Lambda_{1}$ the projection of the set $Z$ into the first coordinate space $\Lambda$; then $\Lambda_{1}$ is analytic, hence a measurable subset of $\Lambda$. Condition (i) implies that $P$ and $Q$ are in $\mathscr{R}(\lambda)$ if $(\lambda ; P, Q) \in Z$; thus, by Lemma 1.1 conditions (ii) and (iii) imply that $\Lambda_{1}=\left\{\lambda \in \Lambda_{0}: B(\lambda) \notin\right.$ $\left.\operatorname{ref}\left(\mathscr{R}^{\prime}(\lambda) \mathscr{S}(\lambda)\right)\right\}$. Since $\mathscr{R}(\lambda)$ is a factor of type $\mathrm{I}_{1}$, II, or III for each $\lambda \in \Lambda_{0}$, we have from $\S 3$ that $\operatorname{ref}\left(\mathscr{R}^{\prime}(\lambda) \mathscr{S}(\lambda)\right)=\mathscr{R}^{\prime}(\lambda) \mathscr{S}(\lambda)$, hence $\Lambda_{1}=\left\{\lambda \in \Lambda_{0}\right.$ : $\left.B(\lambda) \notin \mathscr{R}^{\prime}(\lambda) \mathscr{S}(\lambda)\right\}$. From $B \notin \mathscr{T}$ and $\mu\left(\Lambda \backslash \Lambda_{0}\right)=0$ it now follows that $\mu\left(\Lambda_{1}\right)>0$.

By the principle of measurable choice [14, p. 1041] there exists a measurable mapping $\varphi: \Lambda_{1} \rightarrow \operatorname{Proj}(\mathscr{K}) \times \operatorname{Proj}(\mathscr{K})$ such that $(\lambda, \varphi(\lambda)) \in Z$ for all $\lambda \in \Lambda_{1}$. Then of course $\varphi$ consists of two projection valued measurable mappings $P($ ) and $Q()$ on $\Lambda_{1}$. Extend $P()$ and $Q()$ to $\Lambda$ by defining $P(\lambda)=0$ and $Q(\lambda)=0$ for $\lambda \in \Lambda \backslash \Lambda_{1}$ and denote by $\widetilde{P}$ and $\widetilde{Q}$ projections in $\mathscr{B}(\mathscr{H})$ defined by the two mappings $P()$ and $Q()$. Then condition (i) (together with the fact $P(\lambda)=0$ and $Q(\lambda)=0$ for $\left.\lambda \in \Lambda \backslash \Lambda_{1}\right)$ implies that $\widetilde{P}$ and $\widetilde{Q}$ are in $\mathscr{R}$, (ii) implies that $\widetilde{P} \mathscr{S} \widetilde{Q}=0$, and (iii) implies that $\widetilde{P} B \widetilde{Q} \neq 0$ (since $\mu\left(\Lambda_{0}\right)>0$ ). It follows now that $B \notin \operatorname{ref}\left(\mathscr{R}^{\prime} \mathscr{S}\right)$, but this is in contradiction with the choice of $B$.

The proof of the second inclusion in (4.1) is easier. We must show that each ultraweakly continuous linear functional $\omega$ on $\mathscr{B}(\mathscr{H})$, which annihilates 
$\mathscr{R}^{\prime} \mathscr{S}$, must annihilate $\mathscr{T}$. Let $\omega$ be given by

$$
\omega(T)=\sum_{n=1}^{\infty}\left\langle T x_{n}, y_{n}\right\rangle \quad(T \in \mathscr{B}(\mathscr{H})),
$$

where $\left(x_{n}\right)$ and $\left(y_{n}\right)$ are two sequences of vectors in $\mathscr{H}$ satisfying $\sum\left\|x_{n}\right\|^{2}<$ $\infty$ and $\sum\left\|y_{n}\right\|^{2}<\infty$. For each decomposable operator $T=\int_{\Lambda}^{\oplus} T(\lambda) d \mu(\lambda)$, (4.2) can be rewritten as

$$
\omega(T)=\int \sum_{n=1}^{\infty}\left\langle T(\lambda) x_{n}(\lambda), y_{n}(\lambda)\right\rangle .
$$

(Here the change of order of integration and summation is easily justified by the Fubini theorem, since $\sum\left\|x_{n}\right\|\left\|y_{n}\right\|<\infty$.) Let now $T \in \mathscr{R}^{\prime} \mathscr{S}$. Then $\mathscr{C} T \subseteq \mathscr{R}^{\prime} \mathscr{S}$, hence $\omega(C T)=0$ for all $C \in \mathscr{C}$. Since each $C \in \mathscr{C}$ is a multiplication by some function $f \in L_{\infty}(\Lambda, \mu)$, we see (by replacing $T$ with $C T$ in (4.3)) that

$$
\int_{\Lambda} f(\lambda) \sum_{n=1}^{\infty}\left\langle T(\lambda) x_{n}(\lambda), y_{n}(\lambda)\right\rangle d \mu(\lambda)=0
$$

for all $f \in L_{\infty}(\Lambda, \mu)$. Since $f$ is arbitrary, the last condition is equivalent to

$$
\sum_{n=1}^{\infty}\left\langle T(\lambda) x_{n}(\lambda), y_{n}(\lambda)\right\rangle=0 \quad \text { a.e. }
$$

Since the sums $\sum\left\|x_{n}(\lambda)\right\|^{2}$ and $\sum\left\|y_{n}(\lambda)\right\|^{2}$ are finite almost everywhere (because their integrals are finite), one can define linear functionals $\omega_{\lambda}$ on $\mathscr{B}\left(\mathscr{H}_{\lambda}\right)$ for almost all $\lambda \in \Lambda$ by $\omega_{\lambda}(S)=\sum\left\langle S x_{n}(\lambda), y_{n}(\lambda)\right\rangle$, and by (4.4) we have $\omega_{\lambda}(T(\lambda))=0$ almost everywhere for each $T \in \mathscr{R}^{\prime} \mathscr{S}$. Thus, except possibly on some fixed set of measure 0 , we have $\omega_{\lambda}\left(A_{n}^{\prime}(\lambda) A_{j}(\lambda)\right)=0$ for all $j=1, \ldots, r$ and all $n=1,2, \ldots$. By Lemma 3.1(i) the space

$$
\operatorname{span}\left\{A_{n}^{\prime}(\lambda) A_{j}(\lambda): j=1, \ldots, r ; n=1,2, \ldots\right\}
$$

is ultraweakly dense in $\mathscr{R}^{\prime}(\lambda) \mathscr{S}(\lambda)$ if $\mathscr{R}^{\prime}(\lambda)$ is a factor, hence $\omega_{\lambda}\left(\mathscr{R}^{\prime}(\lambda) \mathscr{S}(\lambda)\right)$ $=0$ almost everywhere. From the definition of $\mathscr{T}$ it now follows $\omega(\mathscr{T})=0$, as claimed. This proves part (i) of the theorem for separable $\mathscr{H}$.

Suppose now that $\mathscr{H}$ is arbitrary, but $\mathscr{R}$ is countably generated. Then for each $x \in \mathscr{H}$ the space $[\mathscr{R} x]$ is separable, hence the range of any countably decomposable projection $E^{\prime} \in \mathscr{R}^{\prime}$ is separable (since, by definition, $E^{\prime}$ can be expressed as a direct sum of a countable set of cyclic projections). Note that $\mathscr{R} E^{\prime}$ has no central portions of type $\mathrm{I}_{n}$ for $n>1$, since $\mathscr{R} E^{\prime}$ is isomorphic to $\mathscr{R} C_{E^{\prime}}$, where $C_{E^{\prime}}$ is the central carrier of $E^{\prime}\left[14\right.$, p. 335]. Let $B \in \operatorname{ref}\left(\mathscr{R}^{\prime} \mathscr{S}\right)$. Then it is easy to verify that $E^{\prime} B E^{\prime} \in \operatorname{ref}\left(E^{\prime} \mathscr{R}^{\prime} E^{\prime} \mathscr{S} E^{\prime}\right)$, hence, by considering $\mathscr{R} E^{\prime},\left(\mathscr{R} E^{\prime}\right)^{\prime}=E^{\prime} \mathscr{R}^{\prime} E^{\prime}, \mathscr{S} E^{\prime}$ and $E^{\prime} B E^{\prime}$ as objects acting on the separable space $E^{\prime} \mathscr{H}$, it follows that $E^{\prime} B E^{\prime} \in \overline{E^{\prime} \mathscr{R}^{\prime} E^{\prime} \mathscr{S} E^{\prime}} \subseteq \overline{\mathscr{R}^{\prime} \mathscr{S}}$. Choosing a net $\left\{E_{\beta}^{\prime}\right\}$ of countably decomposable projections in $\mathscr{R}^{\prime}$ converging strongly to $I$, 
we see that $B \in \overline{\mathscr{R}^{\prime} \mathscr{S}}$. (We remark that the proof of the second part of the theorem is reduced to separable spaces in a similar way. The only difference is that $\overline{E^{\prime} \mathscr{C} E^{\prime} \mathscr{S} E^{\prime}}$ is generally not contained in $\overline{\mathscr{C S}}$ (since $E^{\prime}$ is not necessarily in $\mathscr{C}$ ). But using the isomorphism $\mathscr{R} C_{E^{\prime}} \rightarrow \mathscr{R} E^{\prime}$, it follows that $B C_{E^{\prime}} \in$ $\overline{C_{E^{\prime}} \mathscr{\mathscr { S }} \subseteq \overline{\mathscr{C} S}}$ for each $B \in \operatorname{ref}_{\mathscr{R}}(\mathscr{S})$, hence $B \in \overline{\mathscr{C} S}$, as above.)

Remark. If a von Neumann algebra $\mathscr{R}$ on a separable Hilbert space $\mathscr{H}$ contains central summands of type $\mathrm{I}_{m}$ for $m>1$, then $\mathscr{R}$ contains a twodimensional subspace $\mathscr{S}$ such that the space $\overline{\mathscr{R}^{\prime} \mathscr{S}}$ is not reflexive and the space $\overline{\mathscr{C S}}$ is not $\mathscr{R}$-reflexive. To see this we may assume (using the central decomposition of $\mathscr{R}$ and $\mathscr{R}^{\prime}$ ) that $\mathscr{R}$ is of type $\mathrm{I}_{m}$ and $\mathscr{R}^{\prime}$ is of type $\mathrm{I}_{n}$ for some cardinals $m$ and $n$, where $m>1$. Then $\mathscr{R}$ is (unitarily equivalent to) the von Neumann algebra $L_{\infty}\left(\Lambda, \mathscr{B}(\mathscr{K})^{(n)}, \mu\right)$ consisting of all multiplications by essentially bounded measurable mappings from $\Lambda$ to $\mathscr{B}(\mathscr{K})^{(n)}$ acting on the Hilbert space $L_{2}\left(\Lambda, \mathscr{K}^{(n)}, \mu\right) \cong L_{2}(\Lambda, \mu) \otimes \mathscr{K}^{(n)}$, where $\mathscr{K}$ is a Hilbert space of dimension $m, \Lambda$ is a (complete, separable, compact) metric space, and $\mu$ is a (regular) positive Borel measure on $\Lambda$. Note that $\mathscr{R}^{\prime}=L_{\infty}\left(\Lambda, M_{n}\left(\mathbb{C}_{\mathscr{K}}\right), \mu\right)$ and $\mathscr{C}=L_{\infty}(\Lambda, \mu) I_{\mathscr{K}}^{(n)}$. Let $\mathscr{T}$ be a two-dimensional nonreflexive subspace of $\mathscr{B}(\mathscr{K})$, and let $\mathscr{S}$ be the subspace of $\mathscr{R}=L_{\infty}\left(\Lambda, \mathscr{B}(\mathscr{K})^{(n)}, \mu\right)$, consisting of constant mappings with values in $\mathscr{T}^{(n)}$ (so that $\mathscr{L}$ is isomorphic to $\mathscr{T})$. Then it is not hard to see that $\mathscr{R}^{\prime} \mathscr{S}=L_{\infty}\left(\Lambda, M_{n}(\mathscr{T}), \mu\right)=\overline{\mathscr{R}^{\prime} \mathscr{S}}$ and $\mathscr{C} \mathscr{S}=L_{\infty}\left(\Lambda, \mathscr{T}^{(n)}, \mu\right)=\overline{\mathscr{C} \mathscr{S}}$. Since $\mathscr{T}$ is not reflexive in $\mathscr{B}(\mathscr{K})$, its preannihilator $\mathscr{T}_{\perp}$ is not generated by operators of rank 1 in $\mathscr{T}_{\perp}[15$, Lemma 2]. Since the preannihilator of $M_{n}(\mathscr{T})$ can be identified with all trace class elements in $M_{n}\left(\mathscr{T}_{\perp}\right)$, it follows that $M_{n}(\mathscr{T})_{\perp}$ is not generated by its elements of rank 1 , hence $M_{n}(\mathscr{T})$ is not reflexive in $\mathscr{B}\left(\mathscr{K}^{n}\right)$. Choose now any $B \in \operatorname{ref}\left(M_{n}(\mathscr{T})\right) \backslash M_{n}(\mathscr{T})$ and let $\widetilde{B}$ be the operator on $L_{2}\left(\Lambda, \mathscr{K}^{(n)}, \mu\right)$ defined by the constant mapping with value $B$, that is $\widetilde{B}(\lambda)=B$ for all $\lambda \in \Lambda$. Clearly $\widetilde{B} \notin \overline{\mathscr{R}^{\prime} \mathscr{S}}$; we claim that $\widetilde{B} \in \operatorname{ref}\left(\mathscr{R}^{\prime} \mathscr{S}\right)$. To see this, let $P$ and $Q$ be any projections in $\mathscr{R}$ satisfying $P \mathscr{R}^{\prime} \mathscr{S} Q=0$. Then $P(\lambda) M_{n}(\mathscr{T}) Q(\lambda)=0$ for almost all $\lambda \in \Lambda$ (by the separability arguments, already used in the proof of Theorem 1.3), hence $P(\lambda) B Q(\lambda)=0$ almost everywhere (since $B \in \operatorname{ref}\left(\mathscr{R}^{\prime} \mathscr{S}\right)$ ) and therefore $P \widetilde{B} Q=0$. By Lemma 1.1 it now follows that $\widetilde{B} \in \operatorname{ref}\left(\mathscr{R}^{\prime} \mathscr{S}\right)$. This proves that $\frac{\mathscr{R} \mathscr{R}^{\prime} \mathscr{S}}{\text { is not reflexive. }}$

Since $\mathscr{T}$ is not reflexive in $\mathscr{B}(\mathscr{K}), \mathscr{T}^{(n)}$ is not reflexive in $\mathscr{B}(\mathscr{K})^{(n)}$. The same reasoning as above now shows that $L_{\infty}\left(\Lambda, \mathscr{T}^{(n)}, \mu\right)$ is not reflexive in $L_{\infty}\left(\Lambda, \mathscr{B}(\mathscr{K})^{(n)}, \mu\right)$ and this means that $\overline{\mathscr{C} \mathscr{S}}$ is not $\mathscr{R}$-reflexive.

The question of relative reflexivity in general $C^{*}$-algebras is of course harder than in von Neumann algebras, but Corollary 2.7 suggests the following

Conjecture. A finite-dimensional subspace of an irreducible $C^{*}$-subalgebra $\mathscr{A}$ of $\mathscr{B}(\mathscr{H})$ is relatively reflexive in $\mathscr{A}$ if and only if it is reflexive in $\mathscr{B}(\mathscr{H})$. 
This conjecture is easily seen to be true if $\mathscr{A}$ contains (one and hence all) nonzero compact operators.

A natural extension of the notion of reflexivity is $n$-reflexivity. A subspace $\mathscr{S}$ of $\mathscr{R}$ is called $n$-reflexive relative to $\mathscr{R}$, where $n$ is a positive integer, iff $\mathscr{S}^{(n)}$ is $M_{n}(R)$-reflexive. For the algebra $\mathscr{R}=\mathscr{B}(\mathscr{H}), n$-reflexivity has been considered for example in $[5,16]$, where a dual characterization is also given. Note that one can define the relative $n$-reflexivity without mentioning the matrix algebra $M_{n}(\mathscr{R})$, but using elementary operators instead. Namely, a subspace $\mathscr{S}$ of $\mathscr{R}$ is $n$-reflexive relative to $\mathscr{R}$ if and only if $\mathscr{S}$ contains all $B \in \mathscr{R}$ satisfying $\varphi(B)=0$ for all elementary operators $\varphi$ of length at most $n$ with coefficients in $\mathscr{R}$ such that $\varphi(\mathscr{S})=0$. (Here an elementary operator of length at most $n$ with coefficients in $\mathscr{R}$ is of course a map $\varphi: \mathscr{R} \rightarrow \mathscr{R}$ defined by (1.4), where all $A_{i}$ and $B_{i}$ are in $\mathscr{R}$.)

A finite-dimensional subspace $\mathscr{S}$ of $\mathscr{B}(\mathscr{H})$ is $n$-reflexive if and only if $\mathscr{S}^{(n)}$ is reflexive in $M_{n}(\mathscr{B}(\mathscr{H}))=\mathscr{B}\left(\mathscr{H}^{n}\right)$, and this is the case if and only if $\mathscr{S}$ contains all operators $B \in \mathscr{B}(\mathscr{H})$ satisfying $B^{(n)} x \in \mathscr{S}^{(n)} x$ for all vectors $x=\left(x_{1}, \ldots, x_{n}\right) \in \mathscr{H}^{n}$. Denote by $\mathscr{K}_{x}$ the subspace of $\mathscr{H}$ spanned by the components $x_{1}, \ldots, x_{n}$ of $x$. Then the condition $B^{(n)} x \in \mathscr{S}^{(n)} x$ means that $B\left|\mathscr{K}_{x} \in \mathscr{S}\right| \mathscr{K}_{x}$, where $\mathscr{S} \mid \mathscr{K}_{x}=\left\{S \mid \mathscr{K}_{x}: S \in \mathscr{S}\right\}$. Since each $n$-dimensional subspace $\mathscr{K}$ of $\mathscr{H}$ is equal to $\mathscr{K}_{x}$, where $x \in \mathscr{H}^{n}$ is any vector such that its components $x_{1}, \ldots, x_{n}$ form a basis of $\mathscr{K}$, it follows that $\mathscr{S}$ is $n$-reflexive if and only if it contains all operators $B \in \mathscr{B}(\mathscr{H})$ satisfying $B|\mathscr{K} \in \mathscr{S}| \mathscr{K}$ for each $n$-dimensional subspace $\mathscr{K}$ of $\mathscr{H}$. We shall prove that each $n$ dimensional subspace of $\mathscr{B}(\mathscr{H})$ is $n$-reflexive and, more generally, that the space $\overline{\mathscr{R}^{\prime} \mathscr{S}}$ is $n$-reflexive (relative to $\mathscr{B}(\mathscr{H})$ ) for each von Neumann algebra $\mathscr{R}$ on $\mathscr{H}$ and each $n$-dimensional subspace $\mathscr{S}$ of $\mathscr{R}$.

Recall that a subspace $\mathscr{K}$ of $\mathscr{H}$ is separating for a space of operators $\mathscr{S}$ on $\mathscr{H}$ iff the only operator $S \in \mathscr{S}$ satisfying $S(\mathscr{K})=0$ is $S=0$. By [5, p. 36] for each $r$-dimensional subspace $\mathscr{S}$ of $\mathscr{B}(\mathscr{H})$ there always exists an $r$-dimensional separating subspace in $\mathscr{H}$. In the lemma that follows we give a slight improvement of this result. For the proof we need the generalization of Kaplansky's lemma on locally algebraic operators, obtained by Aupetit [4, Theorem 2], which can be formulated in the following way:

If an r-dimensional subspace $\mathscr{S}$ of $\mathscr{B}(\mathscr{H})$ has no separating vectors, then $\mathscr{S}$ contains a nonzero operator of rank $\leq r-1$.

In [4] the result is formulated (in slightly different language) for operators between two arbitrary complex vector spaces and it is in fact valid for vector spaces over arbitrary infinite fields. The next lemma is also true in this more general algebraic setting, but since the generalization requires more or less only notational changes, we remain in the Hilbert space context.

For each $x, y \in \mathscr{H}$ the symbol $x \otimes y$ denotes the rank-1 operator on $\mathscr{H}$ defined by $(x \otimes y) z=\langle z, y\rangle x \quad(z \in \mathscr{H})$. 
Lemma 4.2. Let $\mathscr{S}$ be an $r$-dimensional subspace of $\mathscr{B}(\mathscr{H})$, where $r \geq 2$. Then either there exists an $(r-1)$-dimensional separating subspace $\mathscr{K}$ for $\mathscr{S}$ or $\operatorname{dim}[\mathscr{S H}]=1$.

Proof. Let us first consider the case $r=2$. Assume that there does not exist any 1-dimensional separating subspace for $\mathscr{S}$. Then by the result from [4] quoted above $\mathscr{S}$ contains an operator $A_{1}$ of rank 1 . Choose any $A_{2} \in \mathscr{S}$ linearly independent of $A_{1}$. Then for each $x \in \mathscr{H}$ there is a nontrivial combination $\alpha_{1}(x) A_{1} x+\alpha_{2}(x) A_{2} x=0 \quad\left(\alpha_{j}(x) \in \mathbb{C}\right)$, since $x$ is not separating for $\mathscr{S}$. This implies that $A_{2} x \in A_{1} \mathscr{H}$ for all $x$ such that $\alpha_{2}(x) \neq 0$. On the other hand, if $\alpha_{2}(x)=0$, then $\alpha_{1}(x) \neq 0$ and the same equation implies $A_{1} x=0$, hence $x \in \operatorname{Ker} A_{1}$. Since each $x \in \operatorname{Ker} A_{1}$ can obviously be expressed as $x=x_{1}+x_{2}$, where $x_{1}, x_{2} \notin \operatorname{Ker} A_{1}$, it follows that $A_{2} x \in A_{1} \mathscr{H}$ for all $x \in \mathscr{H}$. Since $\left\{A_{1}, A_{2}\right\}$ is a basis of $\mathscr{S}$, we have $[\mathscr{S} \mathscr{H}]=A_{1} \mathscr{H}$, which is a one-dimensional space.

Suppose now, inductively, that the lemma is true for all subspaces of $\mathscr{B}(\mathscr{H})$ of dimension at most $r$, where $r \geq 2$ is a fixed integer, and let $\mathscr{S}$ be an $(r+1)$-dimensional subspace of $\mathscr{B}(\mathscr{H})$. Assume that there are no separating $r$-dimensional subspaces for $\mathscr{S}$. Then there does not exist any separating vector in $\mathscr{H}^{r}$ for the space $\mathscr{S}^{(r)}$ (since the linear span of the components of a separating vector for $\mathscr{S}^{(r)}$ would be a separating subspace for $\mathscr{S}$ of dimension at most $r$ ). Hence, by the quoted result from [4], $\mathscr{S}^{(r)}$ contains a nonzero operator of rank at most $r$ and, consequently, $\mathscr{S}$ contains an operator $A_{1}$ of rank 1. $A_{1}$ is necessarily of the form

$$
A_{1}=b_{1} \otimes a_{1}
$$

for suitable vectors $a_{1}$ and $b_{1}$ in $\mathscr{H}$, where $\left\|a_{1}\right\|=1$ and $b_{1} \neq 0$. Let $\mathscr{H}_{1}=\left\{a_{1}\right\}^{\perp}$ and let $P$ be the projection onto $\mathscr{H}_{1}$.

Observe that there does not exist any $(r-1)$-dimensional separating subspace for $\mathscr{S P}$ in $\mathscr{H}_{1}$. Indeed, if $\mathscr{K}_{1}$ were such a subspace, then $\mathscr{K}=\operatorname{span}\left\{\mathscr{K}_{1}, a_{1}\right\}$ would be an $r$-dimensional separating subspace for $\mathscr{S}$ (since $a_{1}$ is separating for $\left.\mathscr{S} P^{\perp}\right)$, but this would contradict our assumption about $\mathscr{S}$. This implies that $\operatorname{dim}(\mathscr{S P})=r$; indeed, $\operatorname{dim}(\mathscr{S} P)<r+1$ (since $\left.A_{1} P=0\right)$, and the assumption $\operatorname{dim}(\mathscr{S} P) \leq r-1$ would lead us to the contradiction that $\mathscr{S} P$ has an $(r-1)$-dimensional separating space (by [5]). Moreover, since $\mathscr{S} P$ has no separating spaces of dimension $r-1$, the induction hypothesis implies that $\operatorname{dim}[\mathscr{S} P \mathscr{H}]=1$. If $b$ is a nonzero vector in [ $\mathscr{S} P \mathscr{H}]$, then for each $A \in \mathscr{S}$ the operator $A P$ must be of the form

$$
A P=b \otimes x(A)
$$

for a suitable vector $x(A) \in \mathscr{H}_{1}=P \mathscr{H}$. Since $\operatorname{dim}(\mathscr{S} P)=r$, the space $\mathscr{G}=\{x(A): A \in \mathscr{S}\} \subseteq \mathscr{H}_{1}$ must be also $r$-dimensional. Thus, if $\mathscr{S}_{1}$ is any subspace of $\mathscr{S}$ complementary to $\mathbb{C} A_{1}$ (so that $\mathscr{S}=\mathscr{S}_{1} \oplus \mathbb{C} A_{1}$ ), the mapping $A \rightarrow x(A)$ is one-to-one on $\mathscr{S}_{1}$ and therefore we can choose a basis 
$\left\{A_{2}, \ldots, A_{r+1}\right\}$ for $\mathscr{S}_{1}$ so that $\left\{x\left(A_{2}\right), \ldots, x\left(A_{r+1}\right)\right\}$ is an orthonormal basis for $\mathscr{G}$. Denote $x\left(A_{i}\right)$ by $a_{i}$ and for each $i \in\{2, \ldots, r+1\}$ let

$$
\mathscr{K}_{i}=\operatorname{span}\left\{a_{k}: k \neq i, k=1, \ldots, r+1\right\} .
$$

Then $\operatorname{dim} \mathscr{K}_{i}=r$. Since $\mathscr{S}$ has no $r$-dimensional separating spaces, there exists a nonzero $B_{i} \in \mathscr{S}$ such that $B_{i}\left(\mathscr{K}_{i}\right)=0$. With $B_{i}=\sum_{j=1}^{r+1} \alpha_{i j} A_{j}$ (where $\alpha_{i j} \in \mathbb{C}$ ) it follows for $k \neq i, 1$ (by using (4.5), (4.6), and the orthonormality of the set $\left\{a_{1}, a_{2}, \ldots, a_{r+1}\right\}$ )

$$
0=B_{i} a_{k}=\sum_{j=1}^{r+1} \alpha_{i j} A_{j} a_{k}=\alpha_{i k} .
$$

Thus, $\alpha_{i k}=0$ for all $k \neq i, k=2, \ldots, r+1$, hence $B_{i}=\alpha_{i 1} A_{1}+\alpha_{i i} A_{i}$, and we now have (since $a_{1} \in \mathscr{K}_{i}$ )

$$
0=B_{i} a_{1}=\alpha_{i 1} b_{1}+\alpha_{i i} A_{i} a_{1} .
$$

If $\alpha_{i i}$ were 0 , then (4.7) would imply $\alpha_{i 1}=0$, hence $B_{i}=0$, but this is a contradiction. Therefore $\alpha_{i i} \neq 0$ and from (4.7) we have $A_{i} a_{1}=\beta_{i} b_{1}$ with suitable $\beta_{i} \in \mathbb{C}$, hence $\left(A_{i}-\beta_{i} A_{1}\right) a_{1}=0$, for each $i=2, \ldots, r+1$. Denote $S_{1}=A_{1}$ and $S_{i}=A_{i}-\beta_{i} A_{1}$ for $i=2, \ldots, r+1$. Then $\left\{S_{1}, \ldots, S_{r+1}\right\}$ is a basis for $\mathscr{S}$. For $i \geq 2$ we have $S_{i} a_{1}=0$, hence (since $A_{1} P=0$ ) $S_{i}=S_{i} P=A_{i} P=b \otimes x\left(A_{i}\right)=b \otimes a_{i}$. Thus, we now have

$$
S_{1}=b_{1} \otimes a_{1} \quad \text { and } S_{i}=b \otimes a_{i} \quad \text { for } i=2, \ldots, r+1 .
$$

Let finally $\mathscr{K}=\operatorname{span}\left\{a_{1}+a_{r+1}, a_{2}, \ldots, a_{r}\right\}$. Since $\operatorname{dim} \mathscr{K}=r, \mathscr{K}$ is not separating for $\mathscr{S}$, hence there exists a nonzero $S \in \mathscr{S}$ such that $S(\mathscr{K})=0$. With $S=\sum_{i=1}^{r+1} \gamma_{i} S_{i} \quad\left(\gamma_{i} \in \mathbb{C}\right)$ we now have (by (4.8) and the orthonormality of the $a_{k}$ 's) $0=S a_{k}=\gamma_{k} b$ for $k=2, \ldots, r$, hence $S=\gamma_{1} S_{1}+\gamma_{r+1} S_{r+1}$. But then we see from $0=S\left(a_{1}+a_{r+1}\right)=\gamma_{1} b_{1}+\gamma_{r+1} b$, that $b$ is a multiple of $b_{1}$ (otherwise $\gamma_{r+1}=\gamma_{1}=0$ and $S$ would be 0 ). By (4.8) this means that the ranges of all operators $S_{i}$ are spanned by the same vector $b_{1}$, and since these operators form a basis for $\mathscr{S}$, it follows that $\operatorname{dim}[\mathscr{S H}]=1$.

Proposition 4.3. For any r-dimensional subspace $\mathscr{S}$ of a countably generated von Neumann algebra $\mathscr{R}$ on a Hilbert space $\mathscr{H}$ the space $\overline{\mathscr{R}} \mathscr{S}$ is r-reflexive (relative to $\mathscr{B}(\mathscr{H})$ ), and the space $\overline{\mathscr{C S}}$ is r-reflexive relative to $\mathscr{R}$.

Proof. We shall prove only the assertion about $\overline{\mathscr{R}} \mathscr{\mathscr { S }}$, for the proof of the assertion concerning $\overline{\mathscr{C} S}$ is similar. Let us first consider the case $\mathscr{R}=\mathscr{B}(\mathscr{H})$. We must show that $\mathscr{S}$ contains all operators $B \in \mathscr{B}(\mathscr{H})$ satisfying $B|\mathscr{K} \in \mathscr{S}| \mathscr{K}$ for all $r$-dimensional subspaces $\mathscr{K}$ of $\mathscr{H}$. Denote $\mathscr{T}=\operatorname{span}\{B, \mathscr{S}\}$, so that $\operatorname{dim} \mathscr{T}=r+1$. If there exists an $r$-dimensional separating subspace $\mathscr{K} \subseteq \mathscr{H}$ for $\mathscr{T}$, then $B|\mathscr{K} \in \mathscr{S}| \mathscr{K}$ implies $B \in \mathscr{S}$ and the proof is completed. If there are no $r$-dimensional separating subspaces for $\mathscr{T}$, then by Lemma 4.2 , $\operatorname{dim}[\mathscr{\mathscr { H }}]=1$, hence there exist vectors $c \neq 0, b$ and $a_{j}(j=1, \ldots, r)$ in 
$\mathscr{H}$ such that $B=c \otimes b$ and such that the operators $A_{j}=c \otimes a_{j} \quad(j=1, \ldots, r)$ form a basis of $\mathscr{S}$. The fact that $B|\mathscr{K} \in \mathscr{S}| \mathscr{K}$ for each $r$-dimensional subspace $\mathscr{H}$ of $\mathscr{H}$ implies in particular that $B x$ is a linear combination of $A_{1} x, \ldots, A_{r} x$ for each $x \in \mathscr{H}$. If $x$ is orthogonal to all $a_{j}$, then $A_{j} x=0$ for all $j$, hence $B x=0$ and $x$ must be orthogonal to $b$. This implies that $b \in \operatorname{span}\left\{a_{1}, \ldots, a_{r}\right\}$, hence $B \in \operatorname{span}\left\{A_{1}, \ldots, A_{r}\right\}=\mathscr{S}$.

Let us now consider the case of a general (countably generated) von Neumann algebra $\mathscr{R}$ on $\mathscr{H}$. The same reasoning as in the proof of Theorem 1.3 reduces the proof first to algebras acting on separable spaces and then to the case of factors. Thus, we assume that $\mathscr{R}$ is a factor. If $\mathscr{R}$ is of type II or III, then by Theorem 1.3 $\overline{\mathscr{R}^{\prime} \mathscr{S}}$ is even reflexive, hence we many assume that $\mathscr{R}$ is a factor of type I. Then there exists a Hilbert space $\mathscr{K}$, a cardinal $n$, and a subspace $\mathscr{T}$ of $\mathscr{B}(\mathscr{K})$ such that $\mathscr{R}=\mathscr{B}(\mathscr{K})^{(n)}, \mathscr{R}^{\prime}=M_{n}\left(\mathbb{C} I_{\mathscr{K}}\right)$, and $\mathscr{S}=\mathscr{T}^{(n)}$ (as in the proof of Corollary 3.6). Since $\mathscr{T}$ is finite dimensional, it is easy to see that $\mathscr{R}^{\prime} \mathscr{S}=M_{n}(\mathscr{T})=\overline{\mathscr{R}^{\prime} \mathscr{S}}$. By [16, Theorem 2.1] an arbitrary ultraweakly closed subspace $\mathscr{G}$ of $\mathscr{B}(\mathscr{H})$ is r-reflexive if and only if its preannihilator $\mathscr{G}_{\perp}$ in the predual of $\mathscr{B}(\mathscr{H})$ (which is the trace class $\mathscr{C}_{1}(\mathscr{H})$ ) is the closed linear span of operators of rank at most $r$ in $\mathscr{G}_{\perp}$. In our situation $M_{n}(\mathscr{T})_{\perp}$ can be identified with all trace class elements in $M_{n}\left(\mathscr{T}_{\perp}\right)$. Since $\mathscr{T}$ is an $r$-dimensional subspace of $\mathscr{B}(\mathscr{K}), \mathscr{T}$ is $r$-reflexive in $\mathscr{B}(\mathscr{K})$ by the first paragraph of this proof. It follows that $\mathscr{T}_{\perp}$ is the closure of the linear span of operators of rank at most $r$ in $\mathscr{T}_{\perp}$; consequently $M_{n}(\mathscr{T})_{\perp}$ is also the closed linear span of its elements of rank at most $r$.

There are known simple examples of nonreflexive 2-dimensional subspaces of $\mathscr{B}(\mathscr{H})$, but for larger $r$ the following problem seems to be open.

Problem. For each positive integer $r$ determine the smallest $k=k(r)$ such that all $r$-dimensional subspaces of $\mathscr{B}(\mathscr{H})$ are $k$-reflexive.

We have seen above that $k(r) \leq r$, but for large $r, k(r)$ is perhaps considerably smaller than $r$.

To end this paper, let us note that one can define the relative hyperreflexivity for subspaces of von Neumann algebras as in the case of the algebra $\mathscr{B}(\mathscr{H})$. Namely, for each subspace $\mathscr{S}$ of $\mathscr{R}$ and each $B \in \mathscr{R}$ denote by $d(B, \mathscr{S})$ the distance of $B$ to $\mathscr{S}$, put

$$
\delta_{\mathscr{R}}(B, \mathscr{S})=\sup \{\|P B Q\|: P, Q \in \operatorname{Proj}(\mathscr{R}), P \mathscr{S} Q=0\},
$$

and call $\mathscr{S}$ hyperreflexive relative to $\mathscr{R}$ iff there exists a constant $\kappa$ such that

$$
d_{\mathscr{R}}(B, \mathscr{S}) \leq \kappa \delta(B, \mathscr{S})
$$

for all $B \in \mathscr{R}$. The smallest such constant $\kappa=\kappa_{\mathscr{R}}(\mathscr{S})$ is called the constant of $\mathscr{R}$-hyperreflexivity of $\mathscr{S}$. Note that in general $\delta_{\mathscr{R}}(B, \mathscr{S}) \leq d(B, \mathscr{S})$ for each $B \in \mathscr{R}$, and that $\mathscr{S}$ is $\mathscr{R}$-reflexive if and only if $\delta_{\mathscr{R}}(B, \mathscr{S}) \neq 0$ for each $B \in \mathscr{R} \backslash \mathscr{S}$. The idea of hyperreflexivity was introduced by Arveson, who 
showed in [2] that nest algebras are hyperreflexive in $\mathscr{B}(\mathscr{H})$. Kraus and Larson extended the notion of hyperreflexivity from subalgebras to subspaces in $\mathscr{B}(\mathscr{H})$ and observed that each 1-dimensional subspace of $\mathscr{B}(\mathscr{H})$ is hyperreflexive [16]. The author does not know if a similar result holds in general von Neumann algebras, that is, if a hyperreflexive version of part (ii) of Corollary 2.6 is true. In the special case $A=I$, the hyperreflexive version of Corollary 2.6(ii) is indeed true; more precisely, the centre $\mathscr{C}$ of each von Neumann algebra $\mathscr{R}$ is $\mathscr{R}$-hyperreflexive with $\kappa_{\mathscr{R}}(\mathscr{C}) \leq 2$. This follows from the result of Zsido and Gajendragadkar [11] that for each $B \in \mathscr{R}, d(B, \mathscr{C})=\frac{1}{2}\left\|D_{B}\right\|$, where $D_{B}$ is the derivation on $\mathscr{R}$ defined by $D_{B}(T)=B T-T B \quad(T \in \mathscr{R})$, and from the estimate $\left\|D_{B}\right\| \leq 4 \sup \left\{\left\|P^{\perp} B P\right\|: P \in \operatorname{Proj}(\mathscr{R})\right\}$. The last estimate is a consequence of the fact that projections are precisely the extreme points of the positive part of the unit ball of $\mathscr{R}$ (see the computation in [3] or [8, p. 101]). The hyperreflexive version of the first part of Corollary 2.6 is open even in the special case $A=I$; this is the well-known problem of hyperreflexivity of a general von Neumann algebra.

\section{REFERENCES}

1. C. A. Akemann and G. K. Pedersen, Ideal perturbations of elements in $C^{*}$-algebras, Math. Scand. 41 (1977), 117-139.

2. W. Arveson, Interpolation problems in nest algebras, J. Funct. Anal. 20 (1975), 208-233.

3. __ Ten lectures on operator algebras, CBMS Regional Conf. Ser. in Math., no. 55, Amer. Math. Soc., Providence, R. I., 1983.

4. B. Aupetit, An improvement of Kaplansky's lemma on locally algebraic operators, Studia Math. 88 (1988), 275-278.

5. E. A. Azoff, On finite rank operators and preannihilators, Mem. Amer. Math. Soc. No. 64 (1986).

6. E. A. Azoff, C. K. Fong, and F. Gilfeather, A reduction theory for non-self-adjoint operator algebras, Trans. Amer. Math. Soc. 224 (1976), 351-366.

7. J. B. Conway, A course in functional analysis, Springer-Verlag, New York, 1985.

8. K. R. Davidson, Nest algebras, Pitman Research Notes in Math., no. 191, Longman Scientific and Technical, 1988.

9. P. A. Fillmore and J. P. Williams, On operator ranges, Adv. in Math. 7 (1971), 254-281.

10. C. K. Fong and A. R. Sourour, On the operator identity $\sum A_{k} X B_{k}=0$, Canad. J. Math. 31 (1979), 845-857.

11. P. Gajendragadkar, Norm of a derivation on a von Neumann algebra, Trans. Amer. Math. Soc. 170 (1972), 165-170.

12. P. R. Halmos, A Hilbert space problem book, Springer-Verlag, Berlin, 1982.

13. I. N. Herstein, Rings with involution, Chicago Lectures in Math., Univ of. Chicago, 1976.

14. R. V. Kadison and J. R. Ringrose, Fundamentals of the theory of operator algebras, vols. 1, 2, Academic Press, London, 1983, 1986.

15. J. Kraus and D. R. Larson, Some applications of a technique for constructing reflexive operator algebras, J. Operator Theory 13 (1985), 227-236.

16. __, Reflexivity and distance formulae, J. London Math. Soc. 53 (1986), 340-356.

17. D. R. Larson, On similarity of nests in Hilbert space and in Banach spaces, Lecture Notes in Math., vol. 1332, Springer-Verlag, Berlin, 1988, pp. 179-194. 
18. __ Reflexivity, algebraic reflexivity and linear interpolation, Amer. J. Math. 110 (1988), 283-299.

19. D. R. Larson and B. Solel, Nests and inner flows, J. Operator Theory 16 (1986), 157-164.

20. A. I. Loginov and V. S. Sulman, Hereditary and intermediate reflexivity of $W^{*}$-algebras, Izv. Akad. Nauk SSSR 39 (1975), 1260-1273. (Russian)

21. B. Magajna, A system of operator equations, Canad. Math. Bull. 30 (1987), 200-209.

22. W. S. Martindale, Prime rings satisfying generalized polynomial identity, J. Algebra 12 (1969), 576-584.

23. M. Mathieu, Elementary operators on prime $C^{*}$-algebras. I, Math. Ann. 284 (1989), 223244.

24. F. J. Murray and J. von Neumann, On rings of operators, Ann. of Math. (2) 37 (1936), 116-229.

25. G. K. Pedersen, $C^{*}$-algebras and their automorphism groups, Academic Press, London, 1979.

26. V. S. Sulman, On reflexive operator algebras, Mat. Sb. 87 (1972), 179-187. (Russian)

27. M. Takesaki, Theory of operator algebras. I, Springer-Verlag, New York, 1979.

Department of Mathematics, University of LJubljana, JadRanska 19, 61000 LJubljana, YugosLavia 